\title{
E-conciliation vie de travail / vie hors travail : intervenir par le théâtre-forum
}

E-conciliation of working life and private life: Intervening with forum theatre

Vincent Grosjean, Ophélie Morand, Béatrice Cahour et Marc-Éric BobillierChaumon

\section{OpenEdition}

\section{Journals}

Édition électronique

URL : https://journals.openedition.org/activites/6508

DOI : 10.4000 /activites. 6508

ISSN : 1765-2723

Éditeur

ARPACT - Association Recherches et Pratiques sur les ACTivités

Référence électronique

Vincent Grosjean, Ophélie Morand, Béatrice Cahour et Marc-Éric Bobillier-Chaumon, «E-conciliation vie de travail / vie hors travail : intervenir par le théâtre-forum », Activités [En ligne], 18-2 | 2021, mis en ligne le 15 octobre 2021, consulté le 07 avril 2022. URL : http://journals.openedition.org/activites/6508 ; DOI : https://doi.org/10.4000/activites. 6508

Ce document a été généré automatiquement le 7 avril 2022.

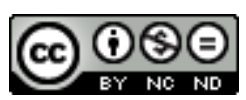

Activités est mis à disposition selon les termes de la licence Creative Commons Attribution - Pas d'Utilisation Commerciale - Pas de Modification 4.0 International. 


\section{E-conciliation vie de travail / vie hors travail : intervenir par le théâtre-forum}

E-conciliation of working life and private life: Intervening with forum theatre

Vincent Grosjean, Ophélie Morand, Béatrice Cahour et Marc-Éric BobillierChaumon

\section{NOTE DE L'ÉDITEUR}

Article soumis le 29/10/2020, accepté le 23/06/2021

\section{Problématique et contexte}

\subsection{Connexion aux outils numériques de communication}

1 La généralisation des équipements de connexion mobile (smartphone, plateformes collaboratives, dispositif de visio-conférence) associés à des outils de communication (messagerie asynchrone ou synchrone de type instantané) et de collaboration (collecticiel, réseaux socio-numériques d'entreprise), a rendu particulièrement prégnante la question de la connexion intense et de la déconnexion aux outils numériques (Boudokhane-Lima \& Felio, 2015 ; Créno \& Cahour, 2016 ; Morand, Cahour, Chaumon, \& Grosjean, 2018). La conciliation de la vie de travail avec la vie hors travail fait partie des enjeux liés à cette évolution technologique à tel point que le législateur français a jugé nécessaire de rendre obligatoire la négociation d'un accord sur le droit à la déconnexion et la régulation des usages des outils numériques afin de préserver la vie familiale et personnelle (article L2242-17 de la loi travail d'août 2016 sur la qualité de vie au travail). Depuis janvier 2017, l'instauration de mesures et la sensibilisation du personnel aux risques liés à des usages immodérés des outils numériques sont devenues 
la règle. Dès avant l'instauration de cette contrainte légale, des tentatives de réponse à cette question avaient été élaborées par les entreprises. Elles ont, dans un premier temps, visé essentiellement à restreindre la connexion d'abord des cadres, puis des salariés fortement connectés, en particulier en dehors des heures normales de travail, le soir et le week-end. C'est ainsi que des chartes, des guides de bon usage, des accords ou encore des formations ont été mis en place dans le but de réguler cette connexion. Les impacts de ces mesures visant à réduire les usages s'avèrent cependant pour le moins mitigés, à la fois en termes d'acceptation de mesures contraignantes et d'effectivité de ces mesures (Prost \& Zouinar, 2015). Ainsi, plusieurs études notent que ces tentatives de régulation ont été souvent suivies de peu d'effets sur les pratiques, en particulier lorsqu'il s'agit de cadres (Boudokhane-Lima \& Felio, 2015 ; Créno \& Cahour, 2016). Parmi les explications de cette faible effectivité des actions visant cette régulation, on peut souligner la position pour le moins ambivalente des cadres par rapport à des solutions normatives de portée générale. On constate en particulier qu'ils souhaitent majoritairement garder la liberté de se connecter pour réguler leur charge de travail et accéder aux informations qu'ils jugent nécessaires pour leur travail, bien que, dans le même temps, ils soient nombreux à dénoncer la lourdeur de leur charge informationnelle ou à s'inquiéter pour la préservation de leur sphère privée (Boudokhane-Lima \& Felio, 2015 ; Morand, Cahour, Chaumon, et al., 2018). En outre, du point de vue du fonctionnement cognitif des personnes faisant un usage extensif de ces technologies, plusieurs auteurs pointent un certain nombre de risques, qui vont d'une réduction de la capacité à prendre des décisions distanciées, due aux sur-sollicitations, à la détérioration de la qualité de la récupération et à des effets sur les capacités attentionnelles (Jauréguiberry, 2014; Lachaux, 2015; Thornton, Faires, Robbins, \& Rollins, 2014 ; Wajcman \& Rose, 2011).

2 Le travail présenté ici porte sur cette problématique de la surconnexion au travail via les outils numériques : dans quelle mesure peut-elle être régulée au sein des collectifs de travail et des organisations, et avec quel outil d'intervention? Il s'inscrit dans le cadre d'une recherche-intervention conduite pendant deux ans en partenariat avec un grand groupe du secteur des télécoms ${ }^{1}$.

3 La finalité principale du travail de recherche-intervention présenté ici se limite à l'exploration des apports d'un dispositif d'échange et de débat sur des pratiques de travail, ici appliqué aux difficultés associées aux usages des outils de communication numériques. Le dispositif d'échange et de mise en débat exploré est le théâtre-forum (cf. 1.4.). Nous nous centrerons ici sur une séquence d'échange et de débat qui a porté plus précisément sur la question de l'équilibre vie de travail/vie hors travail, thème librement choisi par un collectif de professionnels de la santé au travail rassemblé autour de la question de la surconnexion et de ses méfaits. Les apports du dispositif d'échange sont évalués en mobilisant le concept d'intelligence collective (cf. 1.6).

4 En amont de cette recherche-intervention, nous avions réalisé dans ce même groupe industriel un diagnostic des pratiques de connexion posant problème (cf. Morand, 2020). Il existe un coût psychique, cognitif et/ou social induit par l'usage et celui-ci est perçu par les salariés comme problématique.

5 L'intervention avec la méthodologie du théâtre-forum suit et s'appuie sur les résultats de ce diagnostic antérieur réalisé par enquête qui atteste de l'importance des difficultés liées au numérique dans l'entreprise (Claude, Pirlot, Morand, \& Grosjean, 2018; 
Morand, Cahour, Bobillier-Chaumon, \& Grosjean, 2018; Morand, Cahour, Chaumon, et al., 2018).

6 S'agissant de la question des frontières entre sphères de vie, notons que les cadres de cette entreprise, interrogés par questionnaire et par interviews lors de cette enquête, se disent attachés à la flexibilité et que d'aucuns considèrent que certaines chartes professionnelles pourraient avoir pour effet de l'entraver. Cette attitude fait écho à ce qui est parfois qualifié de paradoxe de l'autonomie (Mazmanian, Orlikowski, \& Yates, 2013). Ce terme renvoie aux tensions des professionnels entre leurs souhaits d'autonomie personnelle d'une part et leur volonté de maintenir un haut niveau d'engagement envers leurs collègues et clients d'autre part.

\subsection{La question des frontières vie de travail / vie privée}

7 On considère classiquement que la question de la frontière entre sphères de vie doit être examinée selon deux dimensions (Ashforth, Kreiner, \& Fugate, 2000) :

- La flexibilité, qui renvoie à la capacité, pour chacune des sphères de vie, à réduire ou moduler ses exigences pour s'adapter à une augmentation transitoire des exigences de l'autre sphère ;

- La perméabilité, qui évoque la mesure dans laquelle une frontière poreuse fera que des aspects psychologiques ou comportementaux d'une sphère de vie pourront entrer dans l'autre sphère (Nam, 2014).

8 Ainsi, la sphère de vie professionnelle sera dite flexible si, par exemple, un salarié est en capacité de consacrer à certains moments moins d'énergie psychique au travail lorsqu'il vit des problèmes personnels sollicitants ou importants pour lui, et la sphère de vie privée montrera sa flexibilité si sa famille l'autorise à gérer des soucis professionnels durant son temps de repos, ses week-ends, ses vacances, sans poser de problème. La flexibilité des sphères de vie peut donc être vue comme un bénéfice pour le salarié qui sait s'en servir.

Selon Ashforth (Ashforth et al., 2000) la frontière vie de travail/ vie privée sera dite perméable si des contraintes d'ordre professionnel s'invitent dans la sphère privée sans que la personne concernée puisse maîtriser le phénomène, ou réciproquement ; on peut examiner la perméabilité dans les deux directions. L'interruption et l'intrusion d'une sollicitation d'une sphère de vie dans une autre reflètent donc la perméabilité. Certains auteurs considèrent qu'elle se concrétise dès lors qu'une personne est présente physiquement ${ }^{2}$ dans une sphère et est amenée néanmoins à traiter des problèmes ressortant de l'autre sphère (Clark, 2000).

Flexibilité et perméabilité peuvent être explicites ou implicites, c'est-à-dire découler des politiques affichées et promues par l'entreprise ou découler de pratiques ni débattues, ni supportées par un cadre formel. La flexibilité peut découler de mesures organisationnelles (explicites donc). C'est le cas par exemple des entreprises qui autorisent leurs salariés à donner des heures de travail à des collègues en difficulté, ou à s'absenter pour enfant malade. Elle peut être à l'inverse liée à une règle implicite (par exemple si une baisse de performance liée à une charge familiale accrue demeure peu visible et que les salariés se l'autorisent). De même, la perméabilité peut être structurée par des mesures organisationnelles (Glass, 2000) ou peut tout simplement découler des caractéristiques de la tâche: un ouvrier qui est affecté à une tâche d'assemblage sur une chaine de montage ne risque pas de voir ses contraintes physiques de travail 
déborder sur sa sphère privée. On peut donner comme exemple de mesures organisationnelles engendrant de la perméabilité la mise à disposition d'un téléphone professionnel et la définition de règles d'astreinte. Certains salariés «bloquent » cette perméabilité en ayant un deuxième téléphone (privé) et en éteignant le téléphone professionnel à certaines heures. La perméabilité peut aussi renvoyer à des normes sociales implicites, à une culture métier, à des attitudes personnelles et à des contraintes vécues comme "objectives » de l'activité, du métier, qui vont avoir pour conséquence que de telles stratégies seront perçues comme légitimes ou non et qu'elles seront déployées ou non. Ce sera le cas par exemple de commerciaux qui estiment ne pas pouvoir s'autoriser à ne pas répondre à un de leurs clients qui appelle après $17 \mathrm{~h}$, même après qu'ils ont quitté le bureau.

Figure1 : Typologie de profils régissant les rapports vie privée/vie professionnelle.

Figure 1: Typology of profiles governing the relationship between private and professional life

\begin{tabular}{|l|l|l|}
\hline \multicolumn{1}{|c|}{ Perméabilité } & Forte perméabilité & Faible perméabilité \\
\hline Flexibilité & Intégration & Autonomie \\
\hline Faible flexibilité & $\begin{array}{l}\text { Interférence } \\
\text { LIW (Life Interference with Work) } \\
\text { WIL (Work Interference with Life) }\end{array}$ & Segmentation \\
\hline
\end{tabular}

La littérature considère que le positionnement sur ces deux dimensions de la flexibilité et de la perméabilité génère quatre profils dans la gestion des frontières entre vie professionnelle et vie privée (El-Wafi, Brangier, \& Zaddem, 2016). On peut considérer qu'il y a deux profils où la flexibilité et la perméabilité vont dans le même sens, que nous avons présentés en gros caractères et en italique sur le graphique :

- Intégration lorsqu'on constate des transferts d'affects, de valeurs et de comportements manifestes entre les deux domaines, i.e. de nombreux franchissements des frontières entre vie professionnelle et vie privée (forte flexibilité, forte perméabilité).

- Segmentation lorsque l'individu tend à maintenir les deux sphères de vie aussi cloisonnées que possible, refusant et empêchant que des attentes d'une sphère viennent empiéter sur le temps consacré à l'autre (ni flexibilité ni perméabilité).

Les auteurs (Ashforth et al., 2000 ; Bulger, Matthews, \& Hoffman, 2007 ; Clark, 2000 ; Nam, 2014) y ajoutent classiquement deux profils qui correspondent à une dissociation entre la flexibilité et la perméabilité. Si on les considère du point de vue de la sphère travail, on a alors :

- Autonomie lorsqu'il y a une réactivité forte d'un domaine pour répondre aux exigences de l'autre domaine, mais sans perméabilité. Par exemple, le salarié va pouvoir gérer des charges d'origine privée dans son travail et inversement, en déterminant les niveaux de porosité. C'est alors une configuration de type flexible, mais non perméable.

- Interférence qui renvoie à des irruptions non contrôlées de contraintes liées à un domaine dans l'autre avec une faible flexibilité. C'est par exemple le cas si une forte charge de travail du côté professionnel s'exerce $24 \mathrm{H}$ par jour et 7 jours sur 7 et peut amener un cadre à devoir consacrer tout un dimanche à résoudre un problème de travail de façon subie et non choisie (configuration perméable, mais non flexible). On peut examiner ces interférences dans les deux directions.

Les technologies de la communication auxquelles nous nous intéressons ici, en l'occurrence le couple smartphone-outils de messagerie, ont considérablement modifié 
la flexibilité et la perméabilité des sphères de vie, dès lors que des activités liées à l'une et à l'autre des deux sphères peuvent être réalisées via ces outils numériques, potentiellement en continu et en tout lieu (notion de bureau permanent). Cette évolution a été mise en évidence pour les cadres supérieurs équipés de BlackBerry au début du XXI ${ }^{e}$ siècle (Haddon \& Silverstone, 2000). En 2006, Middleton et Cukier notaient que les utilisateurs de cet équipement avaient adopté le principe de la connexion "permanente et partout " ${ }^{3}$ parce qu'aux yeux de ces cadres cela représentait le moyen le plus efficace pour faire face à leur environnement de travail stressant et surchargé (Middleton \& Cukier, 2006).

14 La généralisation récente des technologies mobiles et du couple smartphone/email a amplifié cette évolution en la diffusant à de larges pans de la population salariée ; cette modification des frontières concerne par conséquent aujourd'hui un nombre croissant de salariés (Wajcman, Bittman, \& Brown, 2008). La littérature consacrée au sujet converge sur le constat que cette généralisation des outils numériques a largement accru la flexibilité et la perméabilité des frontières entre vie de travail et vie de famille ou sphère privée. Avec la disponibilité d'une large palette de dispositifs de communication mobiles (tels le smartphone et la tablette), les salariés ont la possibilité d'adopter désormais des horaires de travail flexibles, qui permettent à leur vie professionnelle et à leur vie familiale de se chevaucher ou de s'intégrer (Valcour \& Hunter, 2005). Dans leur étude sur les employés d'IBM, Hill et al. (Hill, Ferris, \& Märtinson, 2003) constataient dès 2003 que le fait de travailler à partir d'une variété de lieux par le biais des TIC donnait aux employés une grande souplesse dans l'organisation de leurs tâches. Mais ces auteurs notaient déjà que le revers de la médaille était que l'utilisation des TIC pour effectuer le travail pouvait interférer avec leur vie personnelle, accroissant des charges de travail déjà élevées et les conflits travail-famille. "Un appel téléphonique ou un message WhatsApp de son patron ou de ses collègues peut détourner le salarié de sa famille vers les tâches à accomplir, ce qui entraîne l'insatisfaction des membres de la famille » (Leung \& Zhang, 2017).

Dans l'ensemble, la littérature souligne que plus les gens ont l'impression que leur vie est structurée par le biais des TIC, plus ils vivront leur sphère de travail et familiale comme perméables et flexibles (Leung, 2011). Sans surprise, ce sont les personnes bénéficiant d'une grande flexibilité, mais aussi d'une faible perméabilité qui témoignent des niveaux les plus bas de conflit travail-famille (Leung \& Zhang, 2017).

Plusieurs auteurs notent que régulièrement, de nouveaux outils viennent s'ajouter à ceux déjà présents dans l'entreprise (Bobillier-Chaumon, 2017), ce qui fait évoluer la nature des contraintes. C'est ainsi qu'on peut noter l'apparition récente de WhatsApp. Une littérature est en émergence sur des effets psychosociaux plus spécifiquement liés à cette application. Elle souligne comme positif le développement d'un sentiment d'appartenance, d'intimité collective permis par des modes d'interactions spécifiques (Ghliss, 2019 ; O'Hara, Massimi, Harper, Rubens, \& Morris, 2014). L'outil semble en revanche renforcer le brouillage des sphères privée/travail, et le fait qu'il soit adopté par des professionnels sans que l'entreprise n'ait pris la décision de le déployer amène à l'apparition d'usages informels dont les managers n'ont pas nécessairement connaissance (Lebeau, 2017).

Dans le cas des télétravailleurs que la crise sanitaire incite à investiguer tout particulièrement, une étude conduite à Hong-Kong (Leung \& Zhang, 2017) montre que la surcharge informationnelle et le sentiment d'être envahi par la technologie résultent 
de conflits entre le travail et la famille. Ces conflits sont caractérisés par une forte perméabilité de la sphère privée, associée à une faible flexibilité et une faible perméabilité de la sphère professionnelle. Ces résultats sont compréhensibles, car l'utilisation des TIC pour travailler à la maison tend chez les salariés du tertiaire à rendre possible l'envahissement de la vie privée des individus et réduit leur disponibilité pour leur famille, ce qui entraîne une augmentation du stress. Il est intéressant de noter que la perméabilité de la sphère travail face aux contraintes privées est, à l'inverse, négativement liée au technostress, ce qui suggère qu'une frontière perméable vers le domaine professionnel contribue à atténuer le stress, y compris pour des salariés globalement fortement sollicités.

Après ce bref tour d'horizon sur la façon dont la littérature rend compte de la connexion numérique au travail et hors travail et de la structuration des sphères de vie qui en découle, nous allons à présent nous intéresser aux méthodes qui visent à favoriser les échanges autour des pratiques et activités professionnelles, avec un focus particulier sur le théâtre-forum, la méthode retenue pour notre intervention. Le but étant ensuite de déployer cette méthode pour construire un espace d'échange portant sur les pratiques de connexion numérique.

\subsection{Posture réflexive et espaces d'échange en entreprise}

19 Afin de transformer le travail, plusieurs dispositifs d'échange et de mise en débat des pratiques et des normes de travail sont déjà proposés dans la littérature. Un des objectifs premiers de tels dispositifs est de développer une pratique réflexive, en permettant à l'individu d'élargir son point de vue en se confrontant à celui des autres et par ce fait, de créer de nouveaux savoirs collectifs (Casse, 2015; Casse \& Caroly, 2017), un nouveau rapport aux contraintes ou une mobilisation du pouvoir d'agir (Petit \& Dugué, 2011). Dans un contexte de travail, on parle de pratique réflexive collective dès lors que les travailleurs échangent, en prenant leurs propres pratiques comme sujet de discussion, de critique, de pensée, d'élaboration (Begon \& Mairesse, 2013). La question de la pratique réflexive a été mise en avant par Schön (Schön, 1994). Elle prend cependant appui sur les travaux de Piaget sur la prise de conscience et l'abstraction réfléchissante qui constate que l'enfant prend pour objet d'analyse ses propres modes d'action et ses fonctionnements mentaux et essaie de percevoir sa propre façon d'agir. La réflexivité est le mécanisme par lequel le sujet se prend pour objet d'analyse et de connaissance (Rui, 2018). Pour Schön, un double processus est en jeu : la réflexion dans l'action, lorsque le sujet parvient à penser consciemment au fur et à mesure que se déroulent les événements et qui lui permet de réagir lorsque la situation bascule vers quelque chose d'imprévu, et la réflexion sur l'action qui fait référence au fait que le sujet analyse a posteriori ce qui s'est passé et porte un regard critique sur les effets de son action. D'aucuns considèrent qu'elle participe à la construction de l'identité du sujet (Rui, 2018).

En première approximation, la réflexivité est une posture qui permet au sujet d'analyser sa manière d'agir, voire d'expérimenter des modalités d'action susceptibles d'améliorer la qualité et l'efficacité de ses pratiques.

21 Archer (2012) construit une typologie des modes de fonctionnement réflexifs qu'elle applique à nos rapports aux outils numériques; elle envisage trois modes principaux, auxquels elle rajoute un quatrième qu'elle considère dysfonctionnel. 
22 Elle qualifie le premier mode de réflexivité communicante («communicative reflexivity») qui concerne une conversation ouverte impliquant d'autres acteurs (individus ou groupes). Dans ce cas, l'avis explicite de ces acteurs externes et plus globalement les normes et institutions environnantes en vigueur sont importants pour la personne engagée dans ce processus réflexif parce que, selon Archer, elle souhaite être en phase avec ce qui l'entoure. Archer avance que cette forme de réflexivité ne conduit pas à une remise en cause de manières de faire établies collectivement, puisque la personne cherche à ce que ses comportements futurs reproduisent le contexte préexistant (morphostasis).

Le deuxième mode est la réflexivité autonome ("autonomous reflexivity») qui se réfère à un raisonnement mené par l'individu qui évalue seul, au vu de l'ensemble des données qu'il a collectées sur une situation donnée, le meilleur moyen selon lui de résoudre un problème ou de mener une action. Archer et Archer (2003) considère que les acteurs qui mobilisent ce mode réflexif autonome déploient des actions susceptibles de faire évoluer les structures d'action préexistantes (morphogenesis) et donc de conduire à de l'innovation dans les pratiques, ce qui nous intéresse ici dans la mesure où on considère que les pratiques dominantes ne sont pas satisfaisantes.

Le troisième mode est qualifié de méta-réflexivité ("meta-reflexivity») où l'acteur engage en solitaire des conversations internes sur sa propre réflexivité. Il part à la recherche du sens donné à l'univers qui l'entoure et à ses propres actions.

Les personnes engagées dans ce mode méta-réflexif sont, selon Archer les plus susceptibles de remettre en cause l'ordre établi. Elles peuvent s'engager, sur des bases idéologiques, dans des actions « révolutionnaires » qu'on qualifiera maintenant quand il s'agit de nouvelles technologies de disruptives.

26 En ce qui concerne les conversations internes qui ne conduisent pas à la subjectivation de l'action, Archer introduit la notion de réflexivité rompue ( fractured reflexivity ) où l'individu est incapable d'évaluer les situations qui l'entourent, de formuler un avis ou de juger de lui-même ce qui est approprié de faire ou comment il faut agir. Cela peut correspondre aux cas où la réflexivité est entravée, déplacée ou quasi-absente par manque de compétences cognitives, par manque d'accès à l'information, ou plus simplement du fait d'un surinvestissement conduisant à des erreurs de jugement.

Archer (2012) considère en outre que nous vivons dans l'ère du millenium morphogénétique («morphogenetic millenium ») où le digital se diffuse dans bien des domaines de la vie, entraînant des bouleversements sociotechniques inédits et parfois imprévisibles. Ce qu'elle appelle l'impératif réflexif s'impose donc selon elle pour régir les interactions humain-technologie et produire $d u$ changement sociotechnique construit par les acteurs et non subi.

On peut toutefois reprocher à Archer d'aborder la question de la réflexivité essentiellement sous l'angle individuel - y compris pour la réflexivité qualifiée de communicante, puisque la communication consiste à identifier les normes sociales pour s'y conformer - et de ne pas s'intéresser aux dispositifs qui visent précisément à favoriser l'instauration d'une posture réflexive au sein de groupes. Cette auteure aborde la question comme si c'était principalement une question d'attitude personnelle. Or, particulièrement en France, beaucoup de chercheurs ont produit une réflexion théorique sur des façons de rendre fertile le débat sur le travail en vue de le faire évoluer. Cette vitalité française est pour partie expliquée par Van Belleghem 
(2015) par le contexte institutionnel et son insistance sur la création d'espaces d'expression, qui remonte aux lois Auroux instituant un droit d'expression directe et collective pour les salariés en 1984, ce qui a constitué une innovation notable par rapport aux autres pays, malgré son succès plus que mitigé (Linhart, 2019).

S'agissant de l'animation collective de la pratique réflexive, une possibilité consiste à instaurer un espace de discussion ou un dialogue collectif et régulier qui pourra par exemple être animé par le manager (Detchessahar, Gentil, Grevin, \& Stimec, 2015). La tradition ergonomique francophone centrée sur l'activité amène de nombreux exemples où c'est, non pas le manager, mais un intervenant, ergonome ou chercheur, qui mène les échanges. Van Belleghem (2016) s'inscrit clairement dans la tradition ergonomique en considérant comme essentiel dans la constitution du dispositif de s'assurer que les conditions soient réunies pour que les échanges soient bien ancrés dans le réel de l'activité. Concernant le processus de dialogue proprement dit, Van Belleghem $(2016,2018)$ insiste sur le fait que la discussion doit opérer en deux phases, plus précisément doit reposer sur un «double mouvement »: un premier mouvement où le participant énonce sa représentation individuelle, la structure et la spécifie, et un mouvement d'argumentation critique portant sur les représentations individuelles rendues publiques, obligeant le cas échéant le participant à justifier la vision qu'il a offerte à l'analyse des autres. C'est donc bien d'un débat sur le travail et la façon dont il est réalisé qu'il s'agit.

La question des objectifs sous-tendus par le dispositif d'échange est bien souvent en lien avec celle des supports utilisés. Ainsi, (Garrigou, 2011) associe-t-il dans les échanges qu'il mène des vidéos de l'activité et des mesures physiologiques synchronisées avec ces vidéos, comme la fréquence cardiaque. On comprend que cela sert particulièrement l'objectif de mise en débat des pratiques sécuritaires (suis-je dans le rouge du point de vue cardiaque lorsque je fais tel geste dans tel contexte ?) afin de les faire évoluer. Certains auteurs comme Van Belleghem (2016) mettent en avant comme objectif formel de réguler les difficultés de travail au quotidien, ce qui implique selon lui de construire les échanges autour de l'activité réelle passée, alors que pour d'autres la finalité sera de fiabiliser le travail (s'appuyant sur le Retour d'Expérience), ce qui explique que les échanges se centrent sur l'analyse de traces objectives de ce qui fonctionne mal avec, par exemple, l'analyse d'incidents critiques (Casse, 2015). Le cas échéant, le produit de sortie de tels échanges sera de créer de nouvelles normes de travail ou de solliciter la hiérarchie pour qu'elle en élabore (Ibid.).

Si on prend le cas de l'auto-confrontation croisée, développée notoirement par clot et ses collègues s'inscrivant dans le courant de la clinique de l'activité (Clot, Faïta, Fernandez, \& Scheller, 2000), l'objet à partir duquel le dialogue s'opère est constitué de scènes réelles de travail filmées et sélectionnées par l'intervenant et l'objectif est lui aussi centré sur les modes opératoires. La finalité est classiquement de faire évoluer l'activité future dans le sens d'une meilleure prise en compte des contraintes vécues par les uns et les autres. Ainsi, on peut noter dans ces études mettant en place des dispositifs d'échange que la variété des supports servant de substrat à ces échanges est importante et qu'elle est en lien étroit avec les objectifs poursuivis.

Le périmètre du collectif mobilisé rapporté dans la littérature varie également, ainsi que la nécessité d'y «impliquer tous les acteurs concernés pour comprendre un phénomène, quels que soient leur position hiérarchique et leur métier» (Casse, 2015 p. 118). Pour Casse, cette large mobilisation est la condition nécessaire à l'élargissement des 
représentations, ce qu'elle explique par le fait qu'il est nécessaire de sortir des "frontières habituelles de l'activité routinière de production ou d'exploitation" pour comprendre les tenants et les aboutissants d'une activité qui devient dysfonctionnelle. En effet, «les difficultés et arbitrages liés au travail peuvent potentiellement avoir leur origine ou leurs solutions à différents niveaux de l'organisation " (ibid. p. 117). À l'inverse, d'autres dispositifs et d'autres auteurs insistent sur la nécessité de travailler avec un collectif homogène, typiquement composé de salariés de la base, soit pour que la parole critique soit plus libre, soit pour que des pratiques qui s'écartent du prescrit puissent être analysées sans crainte du jugement normatif que pourrait porter la hiérarchie, par exemple (Clot, 1999).

Bonnemain (2019, p. 6) insiste sur le fait que l'activité dialogique en auto-confrontation est conflictuelle. Pour lui, les tensions nées de l'échange sont la source du développement : «il ne peut advenir de nouveau dans l'échange que lorsque les professionnels sont placés dans un cadre qui ne renie pas cette structure conflictuelle [...]. C'est le point de départ potentiel d'un nouveau devenir de la pensée [...]. Le chercheur tente de soutenir l'échange autour des différentes manières de faire [...], car il constitue un indice de cette conflictualité en cours, et donc un indice de développements potentiels... » (Bonnemain, 2019). Petit et Dugué (2011) insistent également sur la mise en visibilité puis en débat des conflits de buts pour la santé psychosociale. Toute la tradition de mise en débat issue de l'éducation populaire (community organising en langue anglaise) et en particulier le théâtre-forum prennent appui sur ce même levier consistant à accepter la conflictualité intrinsèque aux rapports sociaux pour construire d'autres manières de faire (Alinsky, 2012 ; Lénel, 2011 ; Morvan, 2011). Une des difficultés est précisément de libérer la parole alors qu'elle met en cause des positions et implique des rapports de pouvoir.

\subsection{Une méthode d'échange issue de l'éducation populaire : le théâtre-forum}

Le dispositif d'échange qui a été implémenté dans le cadre de cette rechercheintervention, à savoir le théâtre-forum, vise aussi une réflexivité, des prises de conscience et une transformation des modalités d'action - ici limitées aux usages professionnels des outils numériques. La méthode mérite d'être resituée dans le cadre plus large des méthodologies dites de l'Éducation Populaire (Sullivan \& Lloyd, 2006). Il s'agit d'un courant issu du milieu de l'intervention sociale qui s'est ensuite diffusé dans le monde du travail (Boal, 1996 ; de Gaulejac, Giust-Desprairies, \& Massa, 2013). La visée initiale est de stimuler des processus d'émancipation individuelle et collective à des fins de transformation sociale. Les racines de la forme moderne le rattachent à l'action du sociologue américain Saul Alinsky, qui a élaboré une méthodologie de mobilisation communautaire, au départ dans les quartiers pauvres de Chicago, avec comme objectif le développement du pouvoir d'agir de différentes communautés marginalisées (Alinsky, 1971; Ginisty, 1977).

Alinsky met en avant qu'il faut travailler à la fois le lien social et le développement du pouvoir d'agir (empowerment dans la langue de l'auteur), l'un alimentant l'autre. L'idée centrale demeure constante dans toute démarche : l'intervenant extérieur (“Community Organizer"4) part des problèmes tels que vécus subjectivement, voire isolément, par les personnes partageant une certaine communauté de destin ${ }^{5}$, pour les accompagner dans une démarche de renforcement du pouvoir d'agir. Le terme américain d'empowerment 
insiste sur la dimension psychologique liée au sentiment de pouvoir influencer son environnement, donc dans les contextes professionnels qui nous intéressent ici, le travail dans ses dimensions opérationnelles, stratégiques, administratives (Ashforth, 1989). La démarche est construite en approfondissant avec les personnes ciblées la lecture des problèmes qui les concernent et en envisageant avec elles des actions transformatrices. Le renforcement de la capacité des individus et des groupes à définir, selon leurs propres normes, leurs propres problèmes - d'abord individuels et autocentrés, puis le cas échéant collectifs - est donc l'élément premier; il précède et soutient l'action à visée transformatrice. Un parallèle nous semble pouvoir être fait avec le double mouvement évoqué par Van Belleghem, que nous avons présenté plus haut.

L'expression des désaccords et des tensions constitue pour des auteurs comme Alinsky (Alinsky, 2012) et Boal (Boal, Mellac, \& Rigot-Müller, 2004) un élément essentiel de la démarche dont l'ambition est de renforcer l'intégration des classes populaires et des moins favorisées dans le jeu démocratique et dans les rapports de pouvoir qui traversent la société.

En France, l'école de la sociologie clinique (de Castro \& Guerrero, 2013 ; de Gaulejac, 2014) s'est emparée de ces méthodes dans une visée de transformation sociale et d'action dans les milieux de travail. Cette école a donné naissance à plusieurs pratiques: l'organidrame ou organiscope (de Castro \& Guerrero, 2013), le théâtre institutionnel (Badache, 2015), les ateliers citoyens (Guerre, 1999) ou encore le théâtremiroir (Comer, 2017) que nous ne détaillerons pas ici pour nous concentrer sur le théâtre-forum, la méthode mobilisée dans le cadre de notre intervention. Notons cependant que les méthodologies théâtralisées, d'une manière générale (dont les formes les plus documentées sont le théâtre-forum et le psychodrame), présentent l'intérêt d'offrir un cadre pour débattre de façon incarnée d'une situation, en mobilisant et en rendant visibles et légitimes les réactions émotionnelles des salariés, et en permettant que le débat se situe également à ce niveau des émotions, bien distinct de celui d'une discussion abstraite classique.

Le théâtre-forum a été pratiqué puis conceptualisé par le Brésilien Augusto Boal dans les années 70-80 et introduit très tôt en France. Boal y voyait durant la dictature au Brésil un dispositif à fort potentiel émancipateur pour les groupes opprimés, ceci à l'époque où un autre Brésilien, Paulo Freire, développait la pédagogie de l'opprimé, envisageant le dialogue comme espace d'apprentissage par et avec le groupe, espace propice au changement social.

Une séance de théâtre-forum part classiquement d'une saynète illustrant une situation d'injustice mettant en présence un ou des opprimé(s) et un oppresseur. Le but de cette courte scène initiale est de poser le problème, de faire émerger un doute, un questionnement par rapport à une réalité vécue par ceux qui expriment une souffrance, une insatisfaction liée à ce qu'ils vivent quotidiennement. La saynète ne doit durer que quelques minutes et se termine par une tension manifeste, sans solution.

Le dispositif prévoit dans un second temps - le temps du forum - d'interpeller un public pour le faire réagir par rapport à ce qu'il vient de voir. Ce public va donc commenter, exprimer ce qu'il ressent et ce qu'il comprend de ce qui lui a été donné à voir, mais il est également sollicité pour monter sur scène et interagir avec les acteurs sur la base d'une proposition alternative, visant par exemple à dépasser les limites de la scène de départ. Les acteurs réagiront à ce qui leur est proposé en cohérence avec les 
personnages qu'ils incarnent. Ces moments au cours desquels des personnes du public montent sur scène sont appelés "remplacements ». Le dispositif du théâtre-forum encourage donc les spectateurs à intervenir pour modifier l'action dramatique en imaginant et en testant d'« autres possibles ». En cela, le spectateur se transforme, selon le terme consacré, en «Spect-acteur » et mobilise très concrètement son pouvoir d'agir pour modifier le cours des choses dont il a d'abord été témoin (Hamel, 2012). S'il considère que la solution doit se jouer ailleurs que sur le lieu de la scène initiale, il peut demander à ce que ce soit cet « ailleurs » qui soit représenté lors du remplacement, les acteurs s'adapteront et transposeront l'action dans cet ailleurs.

41 À côté des acteurs qui ont mis en actes la scène initiale et réagi en cohérence avec les personnages qu'ils incarnent, et des spect-acteurs qui ont réagi verbalement puis par leurs actes lors des remplacements, le dispositif nécessite encore un intervenant, appelé joker. C'est lui qui assure le lien entre acteurs et spect-acteurs, en facilitant les échanges. Mais il a aussi en charge le "lien entre les divers mondes habités par les participants avec qui le Joker est amené à travailler (Linds, 2006) et ce, en les amenant à problématiser les liens entre ce qu'ils vivent au quotidien et les contextes sociaux plus larges dans lesquels leurs expériences se situent » (Hamel, 2012, p. 11).

NB: Si certaines équipes d'intervention mobilisent une équipe d'acteurs pour construire et animer la scène initiale, tant l'école de sociologie clinique que nousmêmes avons opté pour un travail de constitution de la scène avec les salariés de l'entreprise dans laquelle l'intervention est conduite. Aucun professionnel de la scène n'est donc mobilisé. Le seul professionnel qui intervient, pour ce qui nous concerne, est le psychologue-ergonome qui accompagne les salariés dans la construction de la scène et qui anime le forum dans le rôle de joker.

Ce choix de travailler avec des salariés pour concevoir la scène est en forte résonance avec les objectifs et la philosophie de l'éducation populaire. Il permet en effet de disposer d'un double espace d'échange. Un premier espace d'échange " en non-mixité " (sic) réunit des personnes qui sont motivées par le désir de mettre en visibilité un problème dont elles perçoivent tout particulièrement les inconvénients. Cette mise en visibilité est destinée, adressée (second espace) à des personnes qui ont à leurs yeux le pouvoir ou la charge de le résoudre.

Dans le premier espace d'échange, la question du choix des problèmes à traiter, basé sur les expériences vécues, les discussions sur leur pertinence, l'évocation de stratégies, alterneront avec de la mise en actes puisque le produit de sortie des séances doit être une scène prête à être jouée devant un public plus large. L'intervenant-animateur, qui centre son rôle sur la construction des scènes, la prise de confiance en le processus, intervient peu sur le fond des échanges, donc pour ce qui nous intéresse ici sur les problèmes et solutions en lien aux usages du numérique (position basse Althaus, Grosjean, \& Brangier, 2013). Les échanges sont, pour partie du moins, agis et incorporés, dans la mesure où ils sont mis en scène.

Un second espace intervient ensuite, il s'agit du temps de forum. Les échanges ont lieu cette fois en mixité, puisqu'ils mobilisent un public large, incluant des décideurs et des personnes investies par l'organisation d'un pouvoir ou d'une autorité particulière par rapport au problème évoqué dans la scène. C'est eux qu'il s'agit d'interpeller, afin qu'ils prennent davantage conscience des problèmes qui constituent le quotidien problématisé par les personnes qui ont conçu la scène et par ceux qui vivent une réalité proche de celle qui a été représentée. Là aussi, après que la scène a été vue, le débat est 
pour partie agi et incorporé, du fait de la possibilité des remplacements. Le support est concret, puisque constitué de la scène construite et jouée par les salariés qui ont été volontaires pour la présenter.

Du point de vue des promoteurs de la méthode, l'empowerment et la prise de distance réflexive commencent par la délimitation du problème dont les premiers choisissent de se saisir, qui s'opère au moment de la création de la scène.

Les publications ayant déployé cet outil en contexte professionnel n'envisagent pas cette dualité des espaces de débat. Ainsi, les effets organisationnels que décrit Quinlan (Quinlan, Robertson, Urban, Findlay, \& Bilson, 2020) transitent par une évolution des rapports des membres $\mathrm{du}$ groupe restreint aux contraintes qu'ils vivent quotidiennement. En ce sens, notre choix d'investiguer ces deux espaces est novateur. Par la suite, nous parlerons de phase de scénarisation et de phase de forum pour distinguer ces deux temps d'échange avec ces deux objectifs.

Tableau 1 : Les deux espaces d'échange et la nature des activités réflexives mises en œuvre dans la modalité d'utilisation du théâtre-forum retenue par l'école de sociologie clinique et par nous. Table 1: The two exchange spaces and the nature of the reflexive activities performed in our modality of use of forum theatre

\begin{tabular}{|c|c|c|}
\hline & Phase de scénarisation & Phase de forum \\
\hline $\begin{array}{l}\text { Personnes } \\
\text { impliquées }\end{array}$ & $\begin{array}{l}\text { Salariés mobilisés autour d'un problème } \\
\text { qu'ils vivent ou qu'ils connaissent } \\
\text { particulièrement }\end{array}$ & $\begin{array}{l}\text { Salariés et dirigeants ayant une } \\
\text { responsabilité particulière par rapport } \\
\text { au problème soulevé (managers, élus } \\
\text { syndicaux, } \mathrm{RH}, \ldots \text { ) }\end{array}$ \\
\hline Nom du groupe & Groupe restreint & Groupe large \\
\hline Activités & $\begin{array}{l}\text { Choisir la/les saynètes, se préparer à } \\
\text { la/les jouer, se préparer à improviser lors } \\
\text { des remplacements }\end{array}$ & $\begin{array}{l}\text { Voir la saynète, en donner une lecture, } \\
\text { proposer des manières de la dépasser, } \\
\text { les mettre en actes (remplacements) }\end{array}$ \\
\hline $\begin{array}{l}\text { Positions } \\
\text { incarnées }\end{array}$ & $\begin{array}{l}\text { Scénaristes d'un vécu lié au travail (qui } \\
\text { veulent représenter une réalité qu'ils } \\
\text { souhaitent voir changer)-acteurs (qui la } \\
\text { mettent en actes) - acteurs (qui } \\
\text { improvisent en fonction des suggestions } \\
\text { portées par les remplacements) }\end{array}$ & $\begin{array}{l}\text { Spectateurs (qui voient la saynète) - } \\
\text { débateurs (qui s'expriment à propos de } \\
\text { la saynète et de la réalité à laquelle elle } \\
\text { renvoie) - spect'acteurs (qui testent } \\
\text { d'autres manières de faire lors des } \\
\text { remplacements) }\end{array}$ \\
\hline $\begin{array}{l}\text { Rôles de } \\
\text { l'intervenant }\end{array}$ & $\begin{array}{l}\text { Aider à la convergence des } \\
\text { représentations; rassurer sur les } \\
\text { capacités à la représenter sur scène et à } \\
\text { assurer les remplacements (capacité } \\
\text { d'improvisation en cohérence avec les } \\
\text { réalités du travail) }\end{array}$ & $\begin{array}{l}\text { Moduler les échanges, i.e. assurer une } \\
\text { égale écoute de tout ce qui est dit, en } \\
\text { apposant les points de vue sans les } \\
\text { opposer; permettre l'émergence d'une } \\
\text { représentation de la réalité qui dépasse } \\
\text { et intègre les représentations } \\
\text { individuelles }\end{array}$ \\
\hline $\begin{array}{l}\text { Réflexivité } \\
\text { portant sur }\end{array}$ & $\begin{array}{l}\text { Qu'est-ce que je vis? De quoi ai-je été } \\
\text { témoin dans mon travail ? Comment le } \\
\text { représenter au mieux? Quelles sont les } \\
\text { logiques profondes qui animent les } \\
\text { protagonistes qui doivent être incarnés } \\
\text { dans la scène? }\end{array}$ & $\begin{array}{l}\text { Qu'est-ce qu'il m'a été donné de voir? } \\
\text { A quelle réalité du travail est-ce que } \\
\text { cela renvoie? Quels sont les problèmes } \\
\text { de fond sous-jacents? Que pourrait-on } \\
\text { faire pour que cela se passe } \\
\text { autrement? }\end{array}$ \\
\hline $\begin{array}{l}\text { Disposition } \\
\text { privilégiée }\end{array}$ & Cercle & Public en regard du joker \\
\hline
\end{tabular}

49 Par ce dispositif, on structure l'activité réflexive relative aux modalités de communication, aux rapports de pouvoir et aux pratiques collectives, non en en discutant autour d'une table, mais en les représentant par des actes qui seront ensuite vus, tout comme seront traduites en actes les éventuelles propositions de solution. S'agissant de situations de conflit, de tension, c'est aussi l'occasion de se décentrer, de jouer le rôle de celui ou de celle qu'on considère comme étant de la partie adverse, ce qui rejoint les objectifs de décentration des approches systémiques (Althaus et al., 2013). Les utilisations du théâtre-forum en entreprise, si on se limite à celles qui ont donné lieu à des publications, ont porté sur l'organisation du travail, les rapports de 
domination dans l'entreprise et sur les organisations génératrices de harcèlement (de Gaulejac, 2014 ; Quinlan et al., 2020).

Badache, comme les autres chercheurs-praticiens de l'école de la sociologie clinique, considère que le théâtre-forum mobilise sur les plans à la fois individuel et collectif et permet, à partir des représentations pré-existantes, de susciter des réactions, échanges et réflexions menant à la « conscientisation » (au sens de Freire, 2013). Il note en outre que "c'est un outil qui permet d'aller au-delà du savoir lié à telle ou telle thématique. Il questionne les représentations de chaque participant puis du groupe dans une "situation donnée", il facilite l'expression politique dans un cadre sécurisant et ludique.» (Badache, 2019, p. 110).

52 Les utilisations originelles du théatre-forum mettaient en exergue la relation oppresseur-opprimé et ce qui sortait de la phase de forum a parfois été dénommé un peu vite "solution ». Les formes mobilisées en France en particulier par l'école de la sociologie clinique (Badache, 2015, 2019; de Castro \& Guerrero, 2013; de Gaulejac, 2014 ; Guerre, 1999) s'inscrivent comme nous souhaitons le faire dans une lecture en termes de complexité des rapports sociaux (Morin, 2014). Conflits et tensions sont pour nous comme par exemple pour Badache (2019) à considérer comme des résultantes subjectives pour les antagonistes de contraintes objectives produites par les dysfonctionnements des organisations. La démarche prônée est donc bien de partir de ces vécus subjectifs pénibles pour remonter avec les premiers concernés - ceux qui dénoncent ces tensions au quotidien - aux causes organisationnelles et structurelles de ces tensions.

53 Les promoteurs du théâtre-forum, comme ceux qui utilisent d'autres méthodes basées sur l'expression théâtrale, considèrent que les formes de mise en débat ne peuvent aboutir que si elles engagent les participants au-delà de la seule compréhension rationnelle, intellectualisée - fusse-t-elle partagée - des situations. Elles doivent partir des expériences vécues concrètement par les personnes et mobiliser l'individu et le groupe dans les émotions et dans les corps, de sorte que leur parole soit « incarnée » et non pas abstraite et généralisante (Varela, Thompson, \& Rosch, 2016). Selon Edwards \& Blackwood, le passage par l'expression scénique pour traiter de questions en rapport à des tensions vécues au travail contribue aussi à ce qu'une posture dans laquelle le salarié crée se substitue à une posture dans laquelle il a le sentiment de subir (Edwards \& Blackwood, 2017).

54 L'intervention par le théâtre-forum accorde donc une place centrale aux tensions revisitées collectivement et donne une place à l'expérimentation corporelle, incarnée et émotionnelle de solutions, en parallèle aux réponses rationalisées que le discours et les échanges verbaux favorisent. Le théâtre-forum envisage les tensions vécues (leur mise en actes en plus de leur discussion) comme constitutives d'un levier puissant pour construire des changements organisationnels plus intégratifs d'une diversité de perspectives.

55 La dimension d'intelligence collective tient pour nous à la capacité de cette technique à donner toute leur place aux représentations individuelles tout en permettant l'émergence d'une représentation construite collectivement qui les dépasse en intégrant une large part de la complexité de ce qui se joue dans l'organisation. 


\subsection{Posture de l'intervenant}

dynamique de groupe entre personnes hiérarchiquement proches. La disposition spatiale privilégiée est le cercle, favorisant les échanges et symbolisant la relation d'équivalence.

\subsection{Une grille d'analyse mobilisant le concept d'intelligence collective}

Le terme intelligence collective implique que les représentations qui émergent du fait de la mobilisation d'un dispositif d'échange soient pour partie distinctes et plus riches que les représentations individuelles de chacun des membres. Ainsi, Mack (Mack, 2003) définit-il l'intelligence collective comme « une capacité qui, par la combinaison et la mise en interaction de connaissances, idées, opinions, questionnements, doutes... de plusieurs personnes, génère de la valeur (ou une performance ou un résultat) supérieure à ce qui serait obtenu par la simple addition des contributions (connaissances, idées, etc.) de chaque individu » (repris par Olfa Zaïbet [Olfa Zaïbet, 2007], p. 11).

62 L'intelligence collective renvoie dès lors à la capacité d'une communauté ou d'un collectif de réaliser une tâche ou de résoudre un problème de manière plus efficace et efficiente que les individus, grâce à la collaboration, à la confrontation et au partage des connaissances (Malone, Atlee, \& Lévy, 2008 ; Leimeister, 2010).

Dans son sens large, l'intelligence collective est une intelligence partagée ou de groupe qui émerge de la collaboration et de la compétition de plusieurs individus. Le terme «collectif» décrit un regroupement d'individus qui n'ont pas nécessairement les mêmes attitudes, motivations ou points de vue, mais qui travaillent néanmoins 
ensemble pour trouver des solutions à un problème donné qui peut être complexe ou mal défini.

Le terme "intelligence " peut être ici entendu comme la capacité d'apprendre, de comprendre et de s'adapter à une difficulté issue de l'environnement en mobilisant des connaissances (Leimeister, 2010). L'intelligence collective apparaît lorsque des compétences et des connaissances locales et distribuées sont coordonnées pour atteindre un objectif qui concerne le collectif - même si cet objectif n'est pas nécessairement consensuel - (Secundo, Dumay, Schiuma, \& Passiante, 2016).

En termes de processus, les chercheurs qui mobilisent le cadre de l'intelligence collective décrivent un phénomène où, dans des conditions de diversité (des personnes impliquées), d'indépendance (les contributions d'un individu ne sont pas déterminées par celles des autres individus) et d'agrégation (des mécanismes de mise en commun et de traitement des points de vue individuels sont déployés pour faire émerger une synthèse ou une solution collective), des collectifs peuvent parvenir à de meilleurs résultats/solutions que n'importe quel individu du groupe. Les chercheurs et théoriciens de l'intelligence collective vont ainsi par exemple examiner comment la combinaison et l'alternance d'activités centrées sur le partage d'informations, la clarification du problème, la génération puis le tri de solutions... vont contribuer à l'émergence de solutions innovantes (Berditchevskaia \& Bertoncin, 2021) ou comment, dans un processus d'innovation, se combinent pensée divergente, axée sur la génération d'idées par les membres et leur enrichissement par les regards croisés, et pensée convergente, afin le cas échéant de réduire cet ensemble à une seule alternative finalement retenue (Aggarwal \& Woolley, 2013).

La grille d'analyse de l'intelligence collective peut dès lors être mobilisée pour comprendre comment des structures organisationnelles et de gouvernance, des modalités de mise en réseau et des plates-formes technologiques ou encore des modalités de mise en débat ou de fonctionnement collectif peuvent améliorer le flux des échanges et le partage des points de vue afin de générer des solutions, le cas échéant novatrices face à des problèmes complexes ou mal définis (Murty, Paulini, \& Maher, 2010).

C'est dans cette perspective que nous y faisons appel pour appréhender les apports du dispositif d'échange constitué par le théâtre-forum. L'intelligence, i.e. le degré de sophistication des analyses, la façon dont elles transcendent (dépassent tout en les intégrant) les analyses individuelles, nous intéresse tout particulièrement.

Donc pour nous, le cadre évaluatif constitué autour du concept d'intelligence collective est pertinent pour évaluer la contribution d'un dispositif d'échange sur des pratiques professionnelles comme le théâtre-forum.

Bien que les analyses présentées ici ne porteront pas spécifiquement sur le travail de l'intervenant, il faut néanmoins considérer que celui-ci fait partie du dispositif dont nous essayons d'évaluer la contribution. Son attitude, ses mots, ses réactions à ce qui se dit par exemple lors du forum conditionnent en effet le fait que les participants se sentiront libres de dire et d'avancer dans l'élaboration collective. 


\section{Question de recherche}

Comme signalée dans l'introduction, l'intervention suit et s'appuie sur les résultats d'un diagnostic antérieur réalisé par enquête dans la même entreprise, qui atteste de l'importance des difficultés liées au numérique et à la surconnexion dans l'entreprise et des impacts sur la délimitation des frontières entre vie de travail et vie personnelle (Claude et al., 2018; Morand, Cahour, Bobillier-Chaumon, et al., 2018 ; Morand, Cahour, Chaumon, et al., 2018). Les résultats les plus en lien avec le travail réalisé ici sur les frontières entre sphères de vie sont évoqués brièvement à la fin du paragraphe 1.1.

71 La question de recherche centrale de cet article porte sur l'efficacité du théâtre-forum comme dispositif d'échange, examinée ici sous l'angle de l'intelligence collective. Le dispositif du théâtre-forum permet-il aux collectifs qui se sont prêtés à l'exercice de construire des représentations enrichies à la fois des problèmes liés aux usages excessifs du numérique et des voies de solution?

Partant de l'idée énoncée par Van Belleghem (cf.1.3) selon laquelle l'élaboration collective doit procéder en deux temps, un premier centré sur l'explicitation des positions, un second plutôt centré sur la critique et l'élaboration de représentations plus complexes, nous avons voulu documenter ici comment la méthode permet ce double mouvement, comment elle participe d'une intelligence collective en mouvement. En utilisant le terme d'intelligence collective en mouvement, nous voulons insister sur la dynamique des échanges qui se déploient lorsque le dispositif d'échange est mobilisé.

$73 \mathrm{Au}$ vu des avantages mis en avant par les chercheurs, nous souhaitions voir si le théâtre-forum, mobilisé comme dispositif structurant les échanges collectifs autour des difficultés vécues avec le numérique, peut être d'une part, le moyen à la fois de rendre visible et accessible une réalité qui échappe aux méthodes traditionnelles d'observation de l'activité (ici, le travail médiatisé à distance, i.e. depuis la sphère privée, les situations problématiques de connexion hors temps et lieu de travail) et d'autre part, de définir un cadre dialogique favorisant la confrontation d'expériences et la coconstruction de ressources collectives et organisationnelles qui à terme pourraient déboucher sur le développement des règles d'usage acceptables et soutenables. En amont de tels impacts concrets, les échanges structurés par le dispositif que nous avons déployé doivent d'abord être évalués pour leur capacité à construire des représentations riches, porteuses de sens, et à participer d'une prise de recul i.e. d'une inscription dans une posture réflexive par rapport à une réalité complexe et pour partie problématique. Cette question se pose à la fois pour le groupe restreint, dans sa volonté et sa mission d'instruire l'entreprise et ses dirigeants de difficultés vécues par les salariés, et pour le groupe large, au vu notamment des responsabilités managériales des personnes invitées pour l'exercice.

Concrètement, cette dimension d'intelligence collective concerne l'aptitude de l'outil théâtre-forum et du dispositif d'intervention à assister le collectif dans la construction d'une représentation enrichie d'un problème complexe, porteur de sens pour ce collectif, et de l'espace des solutions qui s'offre à lui. L'enjeu de recherche de l'intervention est de tester la capacité des processus interactifs activés par le théâtreforum à permettre aux collectifs de l'entreprise de créer d'importants "quanta » de valeur (Mack, 2003). La question de recherche retenue ici, s'agissant du dispositif d'intervention, porte donc bien sur son aptitude à soutenir l'intelligence collective du groupe. 
L'ambivalence intrinsèque des cadres de l'entreprise, la multiplicité des enjeux pour partie contradictoires font que le problème répond à toutes les définitions de la complexité (Le Moigne, 2008; Morin \& Le Moigne, 1999). Sans une prise en compte collective de cette complexité, nous considérons que des solutions adaptées ne peuvent émerger. Ce travail d'élaboration d'une représentation plus complexe est à voir à la fois comme un préalable et un complément à la mise en place de solutions régulant concrètement les usages.

\section{Méthodologie}

\subsection{Participants et conduite de l'intervention}

75 télécom. Des mesures classiques visant à réguler les usages du numérique y sont déjà en place, plusieurs accords d'entreprise portent sur le sujet. Cependant, l'enquête réalisée préalablement montre que ces accords sont peu suivis et mal connus. Un collectif réunissant médecins du travail et psychologue du travail du service de santé au travail interne à l'entreprise collabore avec nous depuis plusieurs années sur la question spécifique de la surconnexion et un guide de bonnes pratiques est en cours d'élaboration. Les médecins du groupe de travail ont pour pratique régulière d'interroger sur leurs usages du numérique et leur charge de travail les salariés qu'ils rencontrent dans le cadre des visites périodiques. Dans plusieurs secteurs de l'entreprise, la charge de travail est perçue comme élevée. Les cadres de l'entreprise disposent d'un smartphone et d'un abonnement fournis par l'entreprise.

intervention a été conduite par le premier signataire de cet article. Il avait préalablement été formé à la méthode de théâtre-forum ${ }^{6}$ et avait, au moment où la recherche-action s'est déroulée, une bonne expérience de l'animation de séances de théâtre-forum (rôle de joker) et de l'accompagnement de groupes pour la conception des scènes (rôle d'animation du groupe et de formation). Deux autres signataires de cette contribution étaient présentes, sans rôle d'animation lors de la phase de forum, et une lors des trois séances de la phase de scénarisation.

Comme annoncées au $\$ 1.4$, deux catégories de participants sont à considérer :

- Un premier groupe appelé par la suite "groupe restreint " est constitué de volontaires qui sont d'accord pour se former au rôle d'acteur, pour créer la ou les scène(s) et la/les présenter au groupe large. Notre dispositif prévoyait qu'ils soient recrutés sur la base de leur intérêt pour la question de la connexion numérique et de ses effets sur la qualité de vie et sur les conditions de travail. Dans le cadre de l'intervention relatée ici, les volontaires étaient issus du collectif de médecins et psychologues du travail mobilisé sur le sujet du numérique.

- Un second groupe appelé par la suite "groupe large " va être spectateur de la saynète préparée par le groupe restreint. Ce second groupe a été constitué à notre demande par la Direction RH d'une entité du groupe. Conformément aux objectifs du théâtre-forum, nous avons demandé à ce qu'il soit constitué de personnes concernées par le problème et qu'il inclue des décideurs en position d'initier une action de transformation de la situation de travail ou des pratiques émergentes dénoncées comme problématiques (management supérieur, cadres RH...). 

provenant de différents établissements situés en France. Les disponibilités des uns et des autres ont fait que 9 étaient présents à la première séance, 8 lors des séances 2 et 3 . Le groupe comptait 9 femmes et un homme dont 8 médecins, une personne avec un profil ressources humaines présente uniquement à la première séance et une psychologue.

Le groupe large a rassemblé des cadres de différents niveaux hiérarchiques et RH d'une branche de l'entreprise spécialisée dans le développement de solutions informatiques ; le groupe large incluait aussi des membres du groupe restreint n'intervenant pas dans la scène. 21 personnes au total étaient présentes, dont 12 femmes.

\subsection{Déroulé de l'intervention - deux périmètres d'échange}

Figure 2 : Déroulé théorique et cas étudié d'une séquence d'intervention. Figure 2: Theoretical and implemented sequence of intervention

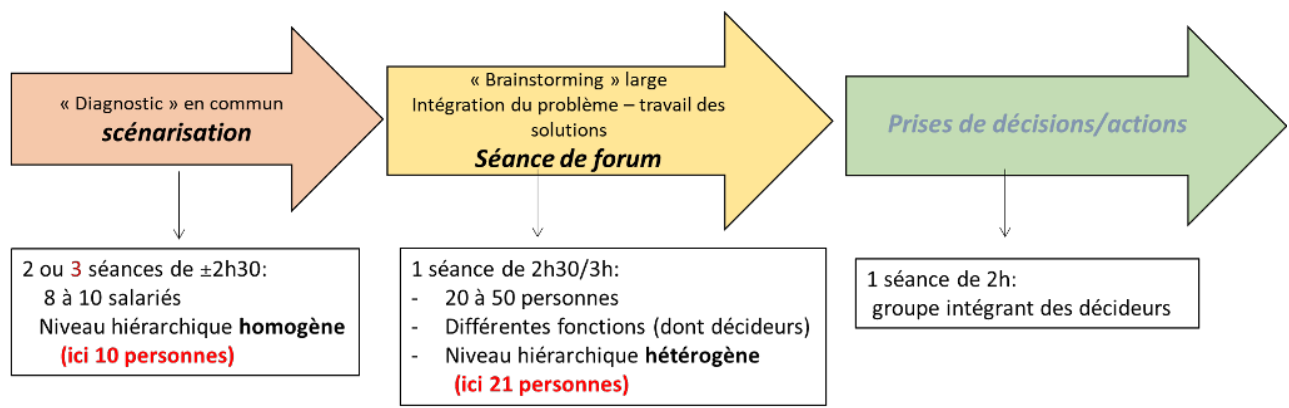

Pour la phase de scénarisation, le temps de mobilisation du groupe restreint annoncé était de trois séances de $2 \mathrm{~h} 30$ pour la préparation de la scène, plus la séance de présentation devant un public, annoncée pour une durée plus importante. Au vu de notre expérience antérieure, cette durée de préparation nous semblait suffisante pour à la fois traiter les questions de fond (« qu'est-ce qui pose particulièrement problème avec les outils numériques?»), construire la scène et se sentir à l'aise avec la perspective des remplacements.

81 Le but assigné aux participants était 1) de construire collectivement une saynète ${ }^{7}$ illustrant une difficulté particulièrement prégnante dans l'entreprise du point de vue du groupe, liée aux usages du numérique dans l'entreprise et à la surconnexion et 2) de se sentir à l'aise pour la présenter au public large et pour improviser lors des remplacements. Conformément aux attendus du théâtre-forum, l'intervenant n'interfère pas avec les choix du groupe, mais structure les échanges afin de faciliter la convergence.

Le processus d'animation prévoit que les participants testent la scène et le cas échéant l'améliorent sur la base des commentaires générés par le groupe et par l'intervenant qui s'abstient de donner son avis sur le bienfondé du scénario, mais commente par exemple la lisibilité du message pour le public final.

En outre, les trois séances de scénarisation ont systématiquement commencé par un tour d'inclusion et se sont terminées par un tour de clôture ${ }^{8}$, ce qui est une pratique courante dans les interventions inscrites dans l'éducation populaire qui permet à 
l'intervenant de collecter des informations sur les ressentis des participants et ici alimente aussi les objectifs de recherche.

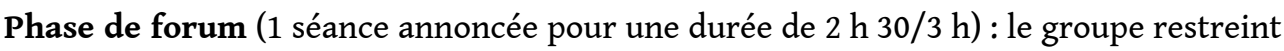
joue la saynète devant le groupe large. Le groupe large travaille sur le mode «forum » avec l'appui de l'intervenant, qui prend le rôle de "joker " (facilitateur). Chacun est sollicité alors pour exprimer: ce qu'il a ressenti, ce qu'il a compris, son avis sur la situation. Des remplacements seront sollicités pour tester le cas échéant des voies de solution. L'intention initiale était de clôturer en envisageant des décisions avec les décideurs, mais cela n'a pas été possible du fait de la durée des échanges et d'un manque de temps. Nous n'aborderons pas cette question des décisions organisationnelles (phase trois de la figure 2) dans le cadre de cette publication.

\subsection{Recueil et analyse de données}

Les supports d'analyse sont liés à trois types de données :

- Les enregistrements audio extensifs (deux enregistreurs audio synchronisés) de toutes les séances et vidéo (caméra haute définition) des scènes jouées nous donnent accès à un matériel bien plus large (environ 12 heures) que celui que nous analysons ici. Les verbatims des séances ont été retranscrits intégralement.

- Un bref questionnaire écrit ( 5 questions ouvertes pour les séances de scénarisation ${ }^{9}$ et 5 pour la phase de forum ${ }^{10}$ ) distribué aux participants à la sortie des deux phases.

- Des entretiens d'explicitation sur le vécu du forum réalisés quelques jours après celui-ci, dans le but de recueillir le vécu des participants et des entretiens individuels et collectifs deux mois après le forum afin de savoir quels changements ont été effectués et perdurent dans le temps. Ces entretiens ont une durée de 20 à 45 minutes et ont été intégralement transcrits.

Dans le présent article, nous centrerons essentiellement l'analyse sur les enregistrements audio et vidéo des séances. Pour la phase de scénarisation, cela permettra de retracer précisément le processus d'élaboration collective de la problématique qui sera retenue pour être présentée, en voyant en quoi ce processus créatif amène à la construction d'une représentation riche et complexe de la réalité vécue. Cela permet de répondre à des questions relatives à l'émergence et la construction des saynètes et à l'intégration en leur sein de problématiques plus ou moins complexes et plus ou moins imbriquées.

Pour ce qui concerne la phase de forum, l'analyse présentée ici, centrée elle aussi principalement sur les verbatims, permettra de retracer la dynamique des échanges, de voir dans quel ordre quels thèmes sont abordés, dans quelle mesure le dispositif permet de traiter des sujets plus ou moins délicats, mettant en évidence des éléments causaux non triviaux, par exemple en lien avec des dimensions politiques, relationnelles et organisationnelles en abordant leur interdépendance, le cas échéant.

Pour ce faire, s'agissant de la phase de scénarisation, nous aborderons les points suivants :

- Reconstitution de la dynamique globale des échanges ;

- Façon dont le choix des thèmes s'opère ;

- Façon dont la scène retenue est construite/sélectionnée ; 
- Articulation des échanges selon six catégories (partage d'expérience, scénarisation, évocation de solutions, réflexion collective, échanges sur la méthode du théâtre-forum, partage d'expérience mixé à de la scénarisation) ;

- Description du scénario finalement retenu / analyse de sa complexité / analyse de sa cohérence par rapport aux intentions débattues.

S'agissant de la phase de forum, nous aborderons les points suivants :

- Dynamique de construction d'une représentation complexe - nature du problème appréhendé - éléments causaux mobilisés dans l'explication de la difficulté figurée par la scène ;

- Choix du remplacement - effet du remplacement sur la richesse de la représentation du problème et des solutions.

\section{Analyses et résultats}

\subsection{Analyse de la construction de la scène (groupe restreint)}

\subsubsection{Choix par les participants de la problématique à présenter en forum}

On peut considérer que le choix de la problématique à présenter lors du forum s'est opéré progressivement lors des deux premières séances de travail en commun et qu'il résulte à la fois de la dynamique au sein du groupe et des choix d'animation.

Partant de la problématique large de la surconnexion et du rapport au numérique, le groupe a travaillé lors de la première séance autour de l'objectif de construire une scène qui concernait les membres du groupe dans le cadre de leur activité professionnelle (prédominance de médecins du travail). C'était donc leur rapport au numérique en tant que salariés de l'entreprise, mais aussi avec des spécificités en lien avec le métier de médecin du travail qui a été le centre des discussions. Plusieurs sujets ont été envisagés - la lecture d'un mail perturbant lors d'une consultation, l'intrusivité du système de chat, le débordement du travail sur la sphère privée. Un thème prégnant lors de cette première séance concernait l'articulation vie de travail/ vie hors travail, mais il en côtoyait d'autres. Lors de la seconde séance, l'intervenant a proposé une focalisation sur la problématique des frontières vie privée / vie professionnelle ce qui a été acceptée. Après que l'option ait été débattue au sein du groupe, le groupe a également décidé de porter sa réflexion sur les difficultés liées au numérique qui impliquent des salariés lambda (et non plus des médecins), i.e. des personnes que les membres du groupe rencontrent dans le cadre de leur activité professionnelle et avec lesquelles ils échangent à propos des difficultés liées au travail en général, ce qui inclut les usages des outils numériques.

Sur la base de cet accord, l'intervenant a proposé de scinder le groupe en trois sousgroupes de trois personnes et chaque sous-groupe a disposé de 15 minutes pour se mettre d'accord sur une problématique. Trois propositions de problématiques, exprimées sous forme d'idées de scène, ont alors émergé et ont été examinées collectivement :

- «Un dimanche en famille»: une salariée veut passer un dimanche avec sa famille, mais va vérifier une présentation à la dernière minute, et puis se rend au parc toujours en famille, mais ne cesse de penser à cette présentation. 
- «Le WhatsApp du Codir»: un nouvel arrivé dans le Codir reçoit un message WhatsApp vers $21 \mathrm{~h}$ et se sent obligé de répondre rapidement avec humour pour paraître « cool et sympa ».

- «Entre deux» : une personne, qui n'arrive pas à travailler en open-space car il y a trop de bruit, décide de rentrer chez elle, mais y est alors sans cesse interrompue par ses enfants.

Le canevas d'intervention prévoyait de leur accorder ensuite 12 minutes pour que les membres du groupe se mettent d'accord pour une seule scène. Ce travail sous contrainte temporelle assez forte a abouti à la proposition d'une scène associant en fait plusieurs sujets: le débordement des contraintes de travail dans la sphère privée (problématique de perméabilité) lors d'un week-end pour une cadre qui aspire à faire partie du collectif Codir et a été récemment intégrée à un groupe WhatsApp ${ }^{11}$ connectant des membres de ce Codir. La scène évoque en outre un moment de convivialité familiale qui pourra être un repas en présence de l'enfant de la famille.

Du point de vue des membres du groupe, la scène associe trois problématiques qui auraient chacune pu être considérée indépendamment :

- Le débordement du travail sur le temps familial ;

- Le désir de se sentir intégré à un collectif pour partie virtuel et pour partie informel (ici matérialisé par WhatsApp) et associé à une forme de valorisation statutaire ;

- L'émergence de perturbations dans les rapports au collectif liées à WhatsApp considéré comme un outil «ambigu » du fait qu'il n'est pas proposé par l'entreprise, mais librement adopté par les salariés (cf. infra).

On voit donc ici illustrée une volonté d'agrégation des idées de chacun qui semble animer le groupe, puisque les deux premières idées de scènes (un dimanche en famille, le WhatsApp du codir) sont reprises et que, si le point lié aux espaces partagés de la troisième ne sera pas exploité, on voit apparaître un enfant dans la scène retenue comme dans l'idée « entre-deux ».

La scène a été intitulée « un dimanche en famille » par le collectif.

Nous présentons au point suivant quelques traits saillants du processus de construction de cette seconde scène.

\subsubsection{Dynamique d'échange : problématiques et élaboration de la scène}

Les contraintes de longueur ne permettent pas de présenter ici l'ensemble de la dynamique des échanges, nous avons choisi de cibler la présentation sur les échanges qui ont conduit à intégrer dans le scénario final un point particulier, en lien avec les usages de WhatsApp.

La problématique de l'utilisation de WhatsApp est considérée d'emblée par les membres du groupe comme étant «en émergence ": "ce n'était pas un souci il y a quelques mois, on commence à en entendre parler négativement ». Si c'est par rapport à un collectif de direction que la question est d'abord évoquée, elle est ensuite associée à d'autres catégories professionnelles que les médecins connaissent par leur pratique médicale, et l'exemple des commerciaux travaillant en boutique est discuté. Les participants soulignent que les nouveaux usages qui semblent se développer avec cet outil apparaissent liés à une problématique d'inclusion/exclusion dans des collectifs peu formels. Les membres des services de santé échangent alors sur leurs impressions : cet outil, dans certains contextes, a pour effet de définir une frontière symbolique entre «ceux qui font partie du groupe WhatsApp » et ceux qui en sont exclus. Ils convergent pour dire que ce mécanisme d'inclusion/exclusion a une grande valeur de généricité. En 
effet, les membres du groupe soulignent que c'est un besoin de tous de se sentir inclus (universalité du besoin d'appartenance), donc un outil qui frustre certaines personnes par rapport à ce besoin et crée pour eux un sentiment ou une crainte d'exclusion génère un malaise et constitue un problème de santé au travail en lien avec les usages du numérique. Les professionnels de santé se sentent légitimes pour souligner ce problème en l'intégrant à la scène projetée.

Du point de vue des difficultés que cela pose à l'employeur, le groupe converge pour dire que cette problématique en lien avec WhatsApp apparait nouvelle et particulière $\mathrm{du}$ fait que, s'agissant d'une application grand public non promue par l'entreprise, réguler son usage apparaît difficile. Les salariés ne sont pas incités à l'utiliser, ils le font de leur propre initiative, ils peuvent considérer que cela fait partie de leur liberté de construire des groupes affinitaires au sein de la sphère professionnelle et que l'entreprise n'a pas à s'en occuper. On touche donc sur une question de frontière et de perméabilité, ici clairement liée à la nature de l'outil (réseautage social) et à son origine (sphère non professionnelle). Selon les arguments avancés par le collectif, et sans que le terme de perméabilité soit utilisé, l'outil WhatsApp est perçu comme un élément générateur d'une perméabilité pour partie choisie et pour partie néfaste et les possibilités de régulation par l'entreprise apparaissent au premier abord plus que limités.

101 Lors des échanges pour construire la scène, on voit au travers de l'extrait à quel point s'entremêlent des informations se situant sur plusieurs plans. L'analyse montre que les verbatims se répartissent en quatre catégories principales selon notre grille d'analyse : réflexions, solutions, expériences vécues et émotions.

Tableau 2 : Extrait des échanges portant sur l'intérêt d'intégrer la question de whatsApp dans la scène.

Table 2: Excerpt from the exchanges concerning the interest of integrating the whatsApp issue into the scene

1. Animateur : WhatsApp c'est quelque chose de nouveau ? D'émergent ?

$\mathrm{M}$ : ben ouais et ça devient une vraie plaie en fait. REFLEXION ?

$S$ : et ça pose la question... REFLEXION

$\mathrm{J}$ : ils sont en train de trouver des solutions internes, de remplacer par des solutions internes SOLUTION

$T$ : ils ont essayé mais ils y arrivent pas. SOLUTION

S: Plazza/

T: parce que WhatsApp le problème c'est étant donné que c'est un réseau auquel tout le monde met, un réseau social, moi j'ai eu régulièrement des problèmes d'humiliation publique... REFLEXION

8. J : bien sûr

9. T : il y a eu aussi des dénonciations, des mises en lignes, des photos EXPERIENCE VECUE/CONNUE

10. I: Quelle horreur... EMOTION

11. $T$ : compromettantes, enfin des choses comme ça..

12. $\mathrm{J}$ : ça pose vraiment des questions REFLEXION

3. S : et qui sont complétement contre les règlements de sécurité des données REFLEXION

14. N: mais là c'était par des collègues ?

15. T : par des collègues, oui. Par des gens qui sont aussi dans le groupe WhatsApp - EXPERIENCE VECUE C'est quelque chose qu'on peut difficilement maitriser REFLEXION

7. oui parce que c'est pas un outil d'entreprise REFLEXION

18. $\mathrm{M}$ : ils peuvent pas maitriser et puis en même temps c'est un mélange d'un truc privé public où les gens ont l'impression qu'ils sont un peu plus dans le domaine du privé donc ils se permettent des choses qu'ils se permettraient peut-être pas autrement et du coup oui ça mélange un peu les genres mais apparemment, au niveau du Codir le problème c'est que chacun veut surenchérir et se montrer sous son meilleur jour... SOLUTION/ REFLEXION 19. S: le plus réactif possible

En voici quelques exemples:

- Partage d'expériences vécues/connues professionnellement: lorsqu'un membre du groupe rapporte des situations connues par sa pratique professionnelle, en lien aux usages 
de WhatsApp qui justifient pour lui l'intégration de cette problématique pour enrichir la scène. On voit que les échanges amènent à l'expression de réactions émotionnelles («quelle horreur... ») qui marquent le fait que le sujet est problématique pour les membres du groupe qui entendent les témoignages.

- Réflexions collectives : les participants essaient d'analyser cette sous-problématique liée à WhatsApp, visiblement pour comprendre ce qui se joue et pour identifier les enjeux. Dans cette discussion, il s'agit en particulier de comprendre les raisons de l'apparition dans les lieux de travail de "groupes WhatsApp» et les effets psychosociaux positifs (recherchés) mais aussi négatifs associés. Les membres du groupe s'accordent pour dire que les collectifs de travail s'emparent de cette technologie et que cela génère ou cristallise la logique d'inclusion/exclusion évoquée plus haut (\$ 1.4.2). Il y a d'un côté ceux qui sont de fait inclus dans le collectif informel WhatsApp et de l'autre ceux qui ne le sont pas, qui peuvent le cas échéant réaliser tardivement qu'un collectif s'est formé et qu'ils en sont exclus. Les membres du groupe disent que ce phénomène existe dans les boutiques, mais aussi au niveau des directions (d'aucuns rapportent qu'il y a un groupe WhatsApp de membres du comité de direction local). Les membres du groupe font l'hypothèse que cela crée des aspirations à " en être », soit pour de simple motif d'inclusion, soit avec en sus des attentes de promotion ou de valorisation d'image de soi (besoin d'estime). La généricité du problème, dans des secteurs aussi différents que les directions et les services commerciaux, est un argument pour construire une scène intégrant cette problématique d'appartenance très spécifique.

- Solutions : de façon complémentaire et imbriquée dans ces réflexions, on constate dans l'interaction 18 de cet extrait qu'un membre du groupe évoque l'absence de solution simple ( «ils ${ }^{12}$ peuvent pas maîtriser [parce que] c'est un mélange d'un truc public/privé ». Puis, dans la même phrase, viennent deux idées, une relative au sentiment de liberté des usagers en général, l'autre qui précise une problématique propre à une catégorie professionnelle particulière "donc ils se permettent des choses qu'ils se permettraient peut-être pas autrement" [...], « au niveau du Codir le problème c'est que chacun veut surenchérir et se montrer sous son meilleur jour $»$.

103 Dans la suite des échanges relatifs à cette question des usages de WhatsApp, nous avons pu observer la cohabitation de plusieurs éléments. Ont été évoqués des constats de tensions en lien à l'outil WhatsApp : les dénonciations, les photos, le fait que c'est un outil non professionnel donc difficile à réguler. Les membres du groupe parlent alors du problème WhatsApp à un niveau générique. Dans le même temps, le groupe évoque aussi la déclinaison plus particulière de l'utilisation de l'outil par un Codir (se montrer sous son meilleur jour, montrer qu'on est réactif, conforme à la philosophie " agile » dans sa dimension d'extrême flexibilité...), soulignant à nouveau une problématique sous-jacente liée au besoin/désir d'appartenance à un collectif (ici prestigieux, car signant un certain niveau de réussite professionnelle) qui peut pousser à accepter des débordements dans la sphère privée via cet outil. Ce qui est exprimé renvoie à l'évocation de situations connues de l'une ou l'autre, mais aussi à des difficultés organisationnelles pour trouver des solutions («ils ont essayé, mais ils n'y arrivent pas ») et à la construction par le groupe d'un positionnement collectif («quelle horreur!»).

La figure 3 permet de visualiser globalement les alternances et la succession des grandes catégories de thèmes abordés dans les échanges. 
Figure 3 : Répartition chronologique des séquences discursives lors de la seconde séance de préparation de la scène.

Figure 3: Chronological distribution of the discursive sequences during the second session of scene preparation

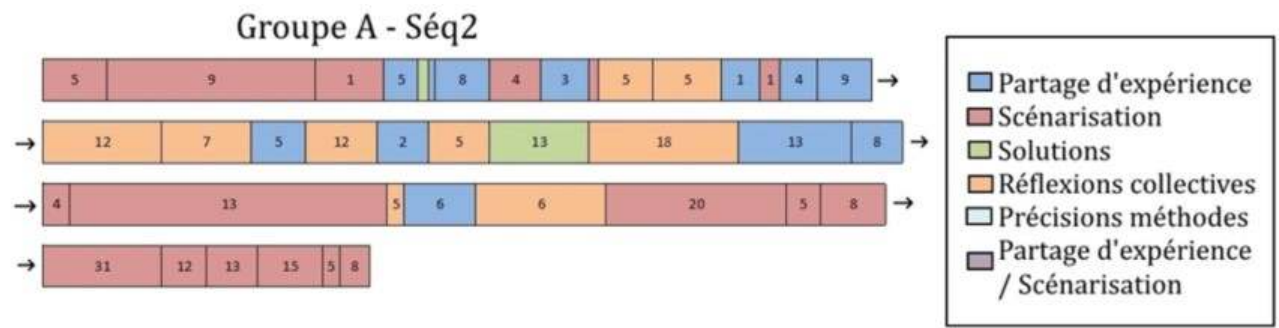

La longueur des segments colorés correspond au nombre de mots, les chiffres renvoient au nombre de tours de parole. Nous pouvons remarquer que la scénarisation est très présente, tout particulièrement dans la seconde moitié de la séquence. Le dispositif donne donc bien une dimension " agie » aux débats, fortement ancrés dans une réalité qu'il s'agit de concrétiser en vue de la jouer. Pendant la séance 1, que nous ne présentons pas ici, la scénarisation constituait la deuxième catégorie la plus fréquente, après le partage d'expérience, ce qui témoigne donc aussi d'un ancrage dans une réalité vécue.

\subsubsection{Description du scénario de la scène retenue}

La contrainte amenée par le cadre (le groupe prépare une scène à animer face à un public) oblige donc à quitter la sphère des affirmations générales abstraites pour catalyser ce que le groupe souhaite exprimer en une scène unique concrète.

L'exemple qui émerge et fait consensus devient celui-ci :

Une cadre (prénommée Stéphanie dans l'histoire) a été récemment incluse dans le groupe WhatsApp de l'équipe de direction (Codir). Elle ressent une certaine fierté à ce qu'elle considère être une marque de reconnaissance de la part de la direction. La scène commence le dimanche à la maison. Elle sait qu'elle a une présentation à faire à la direction le lundi, mais au moment où la scène commence elle est plutôt centrée sur ce qui se passe à la maison : le gouter va commencer avec son fils et son conjoint, puis une balade en forêt est prévue pour un peu plus tard dans l'après-midi. Alors que le goûter est sur la table, elle reçoit des messages WhatsApp d'un membre du groupe Codir. Le fils comme le père montrent par leur attitude qu'ils s'attendaient à ce qu'elle soit disponible pour des activités de loisir en commun (goûter, puis sortie et forêt). Le premier message lui demande où elle en est dans la préparation de sa présentation. La salariée est visiblement tendue suite à ce message, elle se montre déchirée entre son envie d'y répondre et le souci d'être présente, disponible pour son enfant et son conjoint. Elle se met devant l'ordinateur et en vient à demander de l'aide à son conjoint pour avancer plus vite dans la préparation de son exposé. La scène reprend au moment où la famille est en forêt. Alors que son fils s'attend à ce qu'elle soit à présent vraiment disponible pour lui, elle est de nouveau interpellée via le réseau social et s'active sur son téléphone. L'enfant marque de l'énervement et envoie son ballon vers la main de Stéphanie qui tient le téléphone. Celui-ci tombe et se brise, c'est le drame et le ton monte... 
Du point de vue de la correspondance avec les thèmes proposés dans la phase d'émergence des propositions, on voit la recherche d'intégration: la dimension WhatsApp - avec le besoin d'inclusion et la dimension de débordement/porosité « spontanée -, la forte charge de travail au bureau qui se reporte au domicile, le travail du dimanche ont clairement été intégrés dans une seule scène. Les membres du groupe ont également choisi de donner une place dans leur scénario à l'ambivalence du cadre d'entreprise - ici joué par une femme - qui semble vouloir tout combiner, i.e. être irréprochable tant dans la sphère familiale que face aux attentes qu'elle perçoit de sa direction.

109 L'analyse des verbatims, confortée par les entretiens d'explicitation conduits avec les membres de groupe, montre que le choix des problématiques intégrées dans cette scène témoigne d'une volonté de tenir compte de plusieurs critères :

- La gravité du thème : par exemple, une des participantes dit, lors de l'entretien à propos de WhatsApp, « ça devient une vraie plaie »; un autre signale : «j'ai eu régulièrement des problèmes d'humiliations publiques [...] des dénonciations, des mises en ligne de photos compromettantes [...] par des collègues ».

- Le caractère émergent de certaines pratiques, comme WhatsApp : une personne signale qu'en tant que membres d'un service de santé au travail, elle considérait important pour le groupe d'alerter le plus tôt possible sur les risques liés à cette pratique émergente. Aussi, montrer les effets de WhatsApp à une assemblée de décideurs à l'occasion du forum lui semble pertinent.

- La fréquence : certaines personnes ont signalé lors des entretiens que la situation du travail du dimanche était très fréquente : «Je crois qu'on a tous vécu ça».

\subsubsection{Le test du forum sans public}

110 Dans le processus d'apprentissage, des membres du groupe ont, à plusieurs reprises, fait part à l'intervenant de réticences à jouer devant un public réel de leur entreprise, et donc à attirer l'attention des responsables sur un problème par ce canal de la mise en scène, inhabituel pour des professionnels de santé. Les échanges nous ont permis de comprendre qu'une partie de ces craintes étaient liées à la question des remplacements : si la première séance les a convaincus de leur capacité de jouer une scène préparée, la perspective de devoir jouer quelque chose de totalement improvisé en fonction de ce que le public amènerait comme proposition constitue à leurs yeux un réel défi. Afin de les y préparer, l'intervenant a proposé de tester la pratique des remplacements, une partie du groupe qui ne joue pas prenant le rôle du public débattant et suggérant des solutions.

Une première proposition de remplacement a été amenée par une participante qui a suggéré que Stéphanie aurait pu agir en amont et prévenir sa manager qu'elle souhaitait se déconnecter le week-end. La scène à jouer était donc une entrevue entre une remplaçante (issue du public), prenant le rôle de Stéphanie et une des actrices déjà sur scène qui jouerait le rôle de manager de cette dernière.

$\mathrm{Du}$ point de vue de l'intervenant comme des autres membres du groupe, ce remplacement s'est extrêmement bien passé et a été jugé très réaliste ; la manager n'a pas répondu directement aux attentes de Stéphanie, elle a improvisé une manager qui se protégeait en citant les accords d'entreprise (qui donnent effectivement droit à la déconnexion), et en disant que «bien évidemment » ils s'appliquaient. Cependant, elle a 
accompagné cette réponse formelle d'apparence satisfaisante du point de vue réglementaire (droit à la déconnexion) de plusieurs allusions très peu voilées à une "évolution de carrière moins favorable " pour Stéphanie si elle décidait d'instaurer une frontière nettement plus étanche pour isoler sa vie de week-end et à la maison des contraintes liées au travail. Ainsi la personne interprétant le rôle du manager a-t-elle souligné que "d'autres, des collègues, se montraient plus souples » et qu'il serait logique pour elle «d'en tenir compte " pour les promotions et autres marques de reconnaissance.

On voit ici que ce remplacement, construit au sein du groupe restreint sous prétexte de rassurer les membres du groupe sur leur capacité d'improvisation, permet aussi aux participants de mettre en lumière et d'affiner l'analyse de l'expression des normes sociales implicites dans l'entreprise, de la façon dont elles sont le cas échéant " portées " par l'encadrement et acceptées par une majorité silencieuse qui peut très bien n'être que modérément consentante. Ici, "l'analyse succède aux actes" conformément aux présupposés de la méthode. La distance entre règles formelles et pratiques réelles, bien connue des ergonomes (De Keyser \& Olivier, 1972), souligne à quel point l'évolution des pratiques (ici la connexion le week-end via les outils numériques) doit s'accompagner d'une évolution du regard collectif et structurel que l'entreprise favorise par diverses politiques, dans le cas de figure portée par le management, en cohérence ou non avec les accords signés. Les professionnels de santé membres du groupe se montrent ici en capacité «spontanée », et avant toute analyse, d'exprimer par un jeu scénique non préparé ces ambivalences. Cette aptitude a beaucoup réjoui l'animateur (sic).

114 En outre, l'épreuve de la réalité que constitue le remplacement amène le proposant et le public, restreint ici, à constater à quel point la solution proposée (en discuter avec sa cheffe) s'avère peu efficace pour une salariée qui entend ménager sa vie personnelle sans pour autant renoncer à des objectifs de carrière ambitieux.

\subsubsection{Retour des participants}

Nous mentionnons ici les trois points saillants qui ressortent des tours d'inclusion, des tours de clôture et des retours écrits collectés lors de chaque séance.

- La peur de jouer qui s'estompe en changeant de forme. Au début de la première séance, 7 participants sur 10 ont évoqué une forte peur de jouer que certains membres du groupe ont expliqué par le fait de n'être pas à l'aise avec les jeux de rôle en général. «Je déteste les jeux de rôle, je pense que je vais me mettre en retrait » (Bénédicte), " J'ai hésité à venir parce que les jeux de rôles, c'est pas trop ça» (Livia). À la fin de la séance, cette crainte a été exprimée à nouveau, mais en la limitant à la perspective de jouer devant un public élargi. Progressivement, cette crainte plus précise s'est suffisamment estompée pour que chacun soit prêt à s'impliquer face à un public. Nous avions assuré aux participants qu'il était possible de faire appel à des acteurs extérieurs si personne n'avait voulu jouer. Il semble que la perception de la plus-value apportée par des salariés qui connaissent l'entreprise, et seront plus justes, plus fins et réalistes dans les remplacements, a contribué à les rassurer, de même que le cadre, le plaisir du jeu, le climat de bienveillance et de cohésion.

- La création et la cohésion d'un collectif. L'un des effets les plus soulignés par les membres du groupe est l'attachement au groupe et le sentiment d'appartenance qui s'est renforcé au long de ce travail. En effet, lors du tour d'inclusion qui débutait les séances, les participants ont souligné qu'ils étaient heureux de se retrouver et de travailler ensemble. À la fin de la dernière séance, 7 personnes ont noté qu'une des choses les plus appréciées était la 
dynamique et la cohésion de groupe qu'avait permises cette méthode. "On a travaillé ensemble, on s'est exposés, on a joué tous pareils, c'est extrêmement puissant » (Sylvie).

- Mobilisation et coût temporel. Les participants ont apprécié la possibilité de confrontation au réel que permet la méthodologie. En effet, le fait de voir jouer et de jouer leur a permis de se mobiliser davantage qu'à travers un simple discours. Par ailleurs, plusieurs personnes nous ont suggéré d'adapter la méthode au temps de l'entreprise en réduisant les séances de préparation.

\subsection{Analyse du Forum (groupe large)}

\subsubsection{Séquençage du forum}

Après la présentation de la scène, l'échange avec le public a suivi les phases suivantes :

- Premiers échanges et définition du thème.

- Focalisation sur des explications principalement individuelles et familiales.

- Retour à des vécus individuels en lien avec la pression du travail.

- Analyse des causes proches et distantes et apports de solutions par rapport au débordement de charge

- Focalisation sur la personne,

- Focalisation sur le soutien,

- Expression de défiance à l'égard de solutions simples,

- Focalisation sur l'organisation.

- Tentative d'élaboration d'une solution complexe mise en scène lors d'un remplacement.

- Élaborations à partir de ce remplacement.

117 Pour simplifier la lecture, nous avons ajouté des titres résumant le contenu de la discussion. Les participants n'ont pas tous pu être identifiés en termes de fonctions, nous les avons précisées quand nous disposions de l'information et que cela semblait utile à la compréhension.

\section{Premiers échanges et cadrage du thème des échanges}

118 Il apparaît à l'analyse que les premiers échanges semblent jouer un rôle de réassurance quant au cadre d'expression (est-elle libre? Qu'est- ce qui est attendu de nous [public] maintenant que la scène est terminée et qu'on nous donne la parole?)

119 Voici les premiers échanges qui sont intervenus dès la fin de la scène, que nous classons dans cette catégorie : 


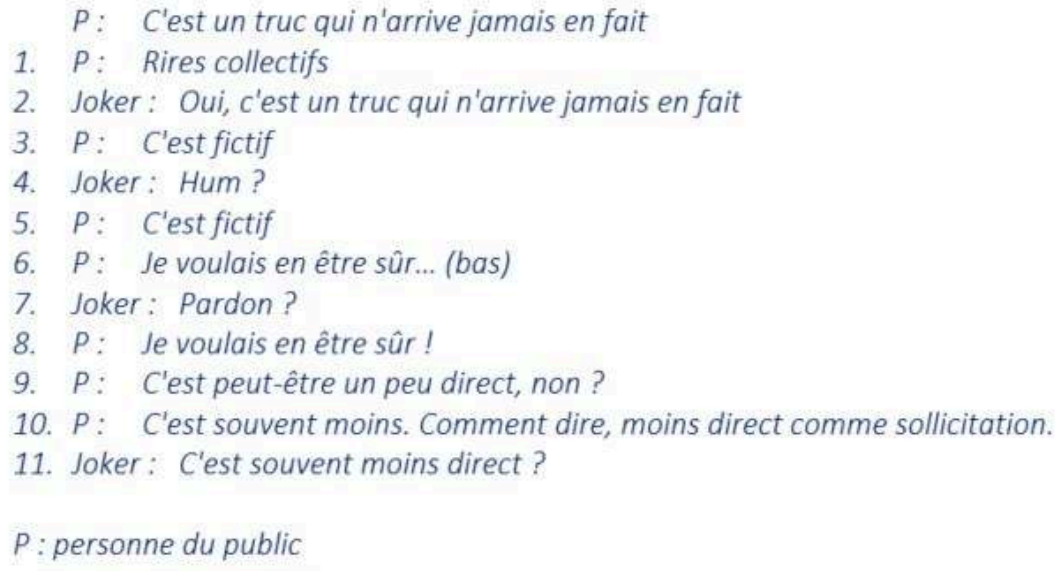

Un participant (manager) dit "C'est un truc qui n'arrive jamais en fait » sur un ton ambigu, laissant penser à une plaisanterie, ce qui fait rire le reste de l'assemblée. Le joker répond en renvoyant l'idée, sans exprimer qu'il souscrit ou non. Un peu après, d'autres personnes émettent l'idée que cela était "un peu direct», i.e. caricatural, avant d'admettre qu'une telle pression peut être vécue, mais plus probablement sans sollicitation du manager («simplement» liée à une motivation intrinsèque de bien faire son travail).

Cette appropriation partielle des thématiques présentes dans ce qui vient d'être vu, qui nous semble presque "négociée " dans ces premiers mots directement adressés au Joker, permet à l'échange de progresser dans un second temps vers des partages de vécus. Les échanges ne vont cependant à aucun moment marquer un intérêt de la part du public pour le vecteur (numérique - WhatsApp) de la sollicitation. Ce qui sera retenu est le fait qu'une salariée soit amenée à travailler le weekend, alors que sa famille la sollicite légitimement pour autre chose. La plausibilité d'une sollicitation directe par le hiérarchique est questionnée également et le collectif en vient à sélectionner comme problématique centrale le travail du week-end, en le mettant en lien dans un premier temps avec ce qui est qualifié de "pression interne " pour évoquer la pression que les salariés ressentent face aux sollicitations du travail. Une concession est faite par rapport aux pressions managériales et externes «Par contre, on reçoit un mail le dimanche, on se dit : bon. Faut quand même que j'le regarde ».

Dans la suite des échanges, une première attitude qui s'exprime tend alors à dire que c'est l'individu qui se met la pression, ou que, comme cela se passe le dimanche, que c'est à la famille de gérer les conflits qui la concernent.

En atteste cet extrait des échanges, où le joker reformule sur un ton neutre :

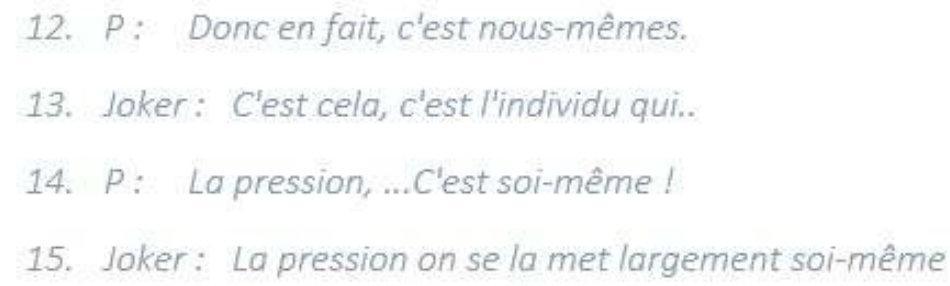


On peut noter également dans ces premiers échanges la centralité du rôle du joker qui acquiesce à tout ce qui est dit, sans rien ajouter ni retrancher.

\subsubsection{Mise en lien de la scène avec des vécus individuels similaires}

Après ces échanges, plusieurs personnes vont témoigner d'un lien entre la scène et un vécu personnel d'une situation proche. Ceci va avoir pour effet de «bloquer » toute possibilité de renvoyer le problème exprimé dans la scène comme étant sans lien avec des causalités professionnelles.

Cette séquence commence de la façon suivante :

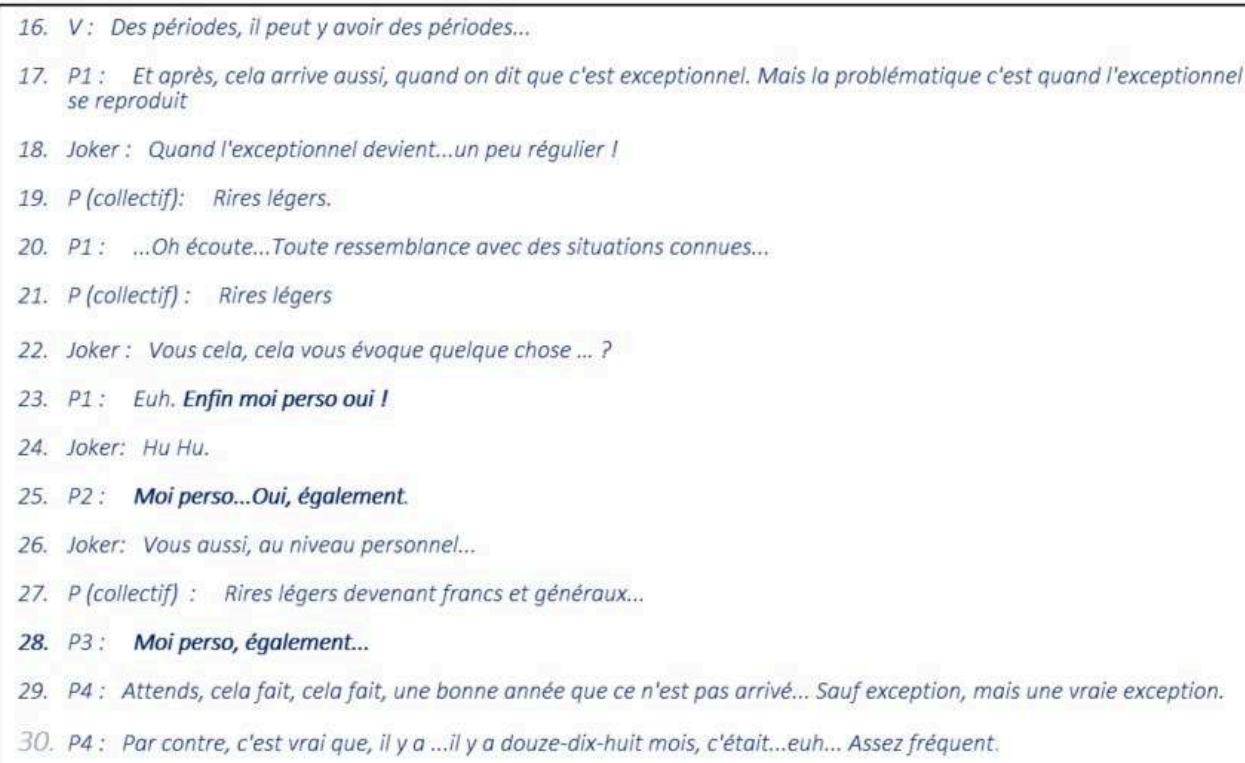

On voit ici que trois personnes - chacune en prenant la précaution de dire qu'il s'agit d'une expérience personnelle avec l'expression «moi perso» qui est reprise par chacune, comme pour sous-entendre que ce n'est pas nécessairement généralisable soulignent que la situation leur parle tant elle fait écho à des situations vécues. Les phrases sont très courtes, très sobres, sans doute parce que la charge émotionnelle est importante, et que ce n'est pas facile de dire cela devant une telle assemblée.

Dans la suite immédiate, quelqu'un rapporte un événement précis (anniversaire d'un enfant) qui l'a amené à gérer un dimanche un problème professionnel complexe («et euh, j'ai passé toute ma soirée au téléphone////reprend son souffle/// casque sur la tête /// blanc///Donc euh, c'est PIRE en fait »). Ce passage un peu haché, rapportant de nouveau une réalité vécue, chargée d'émotions, scelle pour le joker un moment de bascule, à partir duquel va disparaître toute critique portant sur la validité de la scène: puisqu'elle parle sans conteste à quatre salariés du public, qui chacun témoigne à partir de son cas personnel, elle est bien valide! Les échanges ciblent alors comme paramètres problématiques autour desquels vont se concentrer les échanges, d'une part la question de la charge de travail vécue comme non substituable, et d'autre part les possibilités de récupération/repos/prise de distance associées aux impératifs de la sphère privée. En outre, après ces expressions unilatérales (en « je », sans mise en cause de personne) et convergentes, un membre du codir, soit la personne ayant la position hiérarchique la plus élevée dans l'assistance, prend la parole et en vient à valider la centration sur cette charge de travail qui déborde vers la sphère privée en parlant d'une période de 
plusieurs années au cours de laquelle il a été lui-même en astreinte statistique « [...], je peux vous garantir que quand cela dure cinq ans; c'est, c'est vraiment pénible [...] ».

Du point de vue de la dynamique d'empowerment, il nous semble notable que c'est au moment où des salariés témoignent - non sans une certaine émotion - à partir de leur vécu personnel, que la réalité de la question devient incontestable et qu'elle va commencer à être creusée du côté des causes et des solutions possibles dans les échanges.

On voit ici que des contributions individuelles successives extrêmement convergentes ont précipité la bascule des échanges vers quelque chose de très intéressant du point de vue de la prévention des risques psychosociaux et de la mobilisation collective. Comment fait-on lorsqu'on a une responsabilité d'équipe pour conjuguer valorisation de l'investissement professionnel et respect des besoins de récupération? Et lorsqu'on a le statut de cadre, comment conjuguer investissement dans le travail versus préservation de sa vie familiale? Comment doser la charge, éviter qu'elle ne déborde sur le week-end? Évidemment, cette centration n'est pas anticipable par l'intervenant, qui a laissé le groupe restreint choisir le sujet, et ne peut pas contrôler la nature des thèmes qui apparaîtront lors des échanges qu'il va faciliter.

Notons par ailleurs que la thématique portant sur les usages de l'outil WhatsApp, qui faisait partie des messages que le groupe restreint souhaitait faire passer, ne sera pas du tout exploitée par le public, peut-être parce que dans la scène retenue ce n'est qu'un moyen de communication utilisé par le cadre et que WhatsApp n'a pas été perçu comme apportant quelque chose de spécifique vis-à-vis de ce qui devient central : le travail qui déborde.

\subsubsection{Analyse des causes proches et distantes, et apports de solutions}

Figure 4 : Synthèse des principaux éléments causaux abordés. Figure 4: Summary of major causes of the work/family conflict

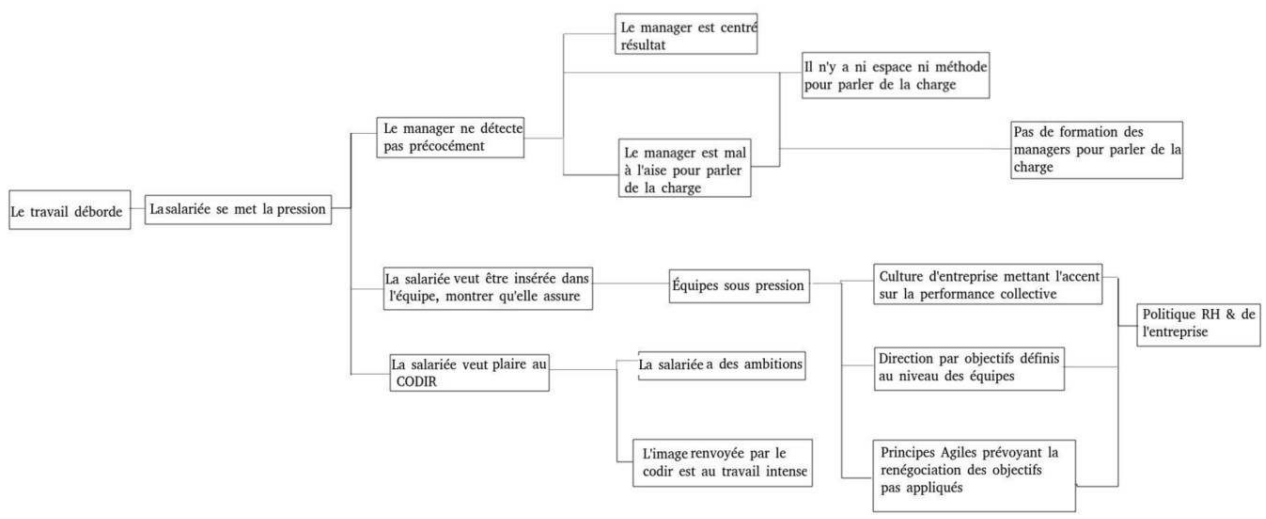

131 La partie centrale de l'échange va à partir de ce moment essentiellement tourner autour de la question de la charge de travail, véhiculée par les outils de communication et rendue possible en tout temps et en tout lieu du fait des outils TIC, que nous résumons en fig. 4. Seront abordés le travail auquel on peut rester connecté $24 \mathrm{~h}$ par jour et 7 jours sur 7 , dès lors que le téléphone et l'ordinateur ne sont pas loin, avec la question des attitudes individuelles et collectives relatives à cette charge, et la façon dont l'organisation et les cultures métiers véhiculent une idéologie de la responsabilité par rapport à des objectifs, mais aussi des principes théoriques selon lesquels les 
travailleurs devraient avoir des moments de répit. Cette partie centrale dure un peu plus de 30 minutes.

Trois sujets de discussion (voir détails plus bas) nous semblent pouvoir être dégagés :

- Détection et action vis-à-vis des personnes qui ressentent une pression excessive et sont donc « en danger ». Interrogation sur qui elles sont, comment on les identifie.

- Moyens d'y faire face à proximité : lieux d'échange, soutien social dans l'équipe, détection des personnes à risque et modalités de soutien managérial.

- Mise en lien du problème avec des modes d'organisation: lien avec la pratique $\mathrm{RH} /$ managériale axée sur l'engagement - la structuration des feedbacks sur la charge au sein des équipes formation des managers aux entretiens axés « charge de travail ».

\section{Détection et action vis-à-vis des personnes qui ressentent une pression excessive}

Le public va considérer qu'il y a des situations où «Le salarié se met la pression ». Le fait que pour certaines personnes, ce fort investissement est conforme à la norme est également invoqué : « on a envie de faire plaisir à l'équipe, parce qu'on a envie d'être content de ce qu'on présente ». Ceci laisse à croire que le premier élément causal évoqué est en lien avec une attitude personnelle. Viennent ensuite des facteurs causaux qui dépassent l'individu, même si c'est exprimé en demandant comment protéger l'individu contre lui-même. La discussion va consister à noter que le management, principalement focalisé sur des indicateurs de performance, a peu de moyens de détecter les situations de surinvestissement, si ce n'est qu'il peut voir que des personnes restent toujours tard le soir par exemple.

Le public soulignera qu'il n'y a ni espace ni moment où ce sujet du risque d'excès de travail pourrait être discuté, et que mettre au point une stratégie face à ce risque n'est pas simple puisque cela impliquerait d'aller à l'inverse de ce qui est encouragé habituellement (i.e. faire pression dans le sens d'un fort investissement de chacun).

\section{Faire parler un acteur}

Dans la palette d'animation dont dispose le joker, on peut voir que «faire parler un acteur " permet de mettre une focale sur le vécu subjectif d'un des personnages de la scène. C'est ce qui a été fait après un échange portant sur : "cela dépend des personnes ", " tout le monde ne vit pas la pression de la même façon", "certains sont capables de prendre conscience de leurs limites et d'autres non ".

L'interpellation de l'actrice a donné lieu à l'échange suivant : 
31. Joker: [interpellant l'actrice jouant le rôle de Delphine] Delphine: Comment est-ce que Delphine vit la situation ? Est-ce qu'elle a l'impression - par rapport [...] à ce qu'on entend ici qu'elle sait se réguler?

32. Réponse de l'actrice: Ben, Delphine elle pense qu'elle sait, euh, elle pense aussi qu'elle se met la pression elle-même, mais, en fait elle est, peut-être aussi un peu le jouet du système, donc eh... C'est cela qui est compliqué, effectivement.

33. Réponse de l'actrice: Hein, c'est-à-dire, qu'entre..., entre ce qu'on..., ce qu'on pense être son propre, son propre arbitre et ce qui finalement n'est pas tant un choix que cela, parfois, Delphine a parfois du mal à faire la différence.

34. P: [le public écoute]

35. Réponse de l'actrice: Peut-être qu'elle aurait pu dire non. Sans doute. Sûrement, il y aurait sûrement pas eu de catastrophe. Maaais, ... Delphine a pas osé ! Parce qu'elle est dans une phase de sa carrière où elle pense que... ben voilà, il faut, il faut y aller quoi ! C'est maintenant. [Petite "exclamation rire"]

Cet acte d'animation du joker consistant à interpeller l'actrice permet d'intégrer à l'échange le point de vue subjectif du «salarié » que le public cherche à aider. Est évoquée la question de l'autonomie réelle de quelqu'un qui a des ambitions de carrière et qui a du mal à se positionner par rapport à des sollicitations qui pourraient empiéter sur la vie privée. Cela écarte des interprétations du type «cela dépend de l'équation individuelle » ou " tout le monde ne réagit pas de la même façon face à la pression ", en mettant en visibilité l'aspiration à être apprécié au sein d'un collectif de travail hiérarchiquement élevé, dès lors qu'on souhaite progresser. L'intention de l'intervenant est ici de faire évoluer la discussion qui aurait pu s'enliser dans une interprétation psychologisante liée aux équations personnelles et dédouanant l'organisation, ceci au moyen du commentaire suivant: "Donc, par rapport à ce cas concret, par rapport à ce que vous disiez, cela vous évoque quelque chose ?... Si on est confronté à une Delphine, - qu'est-ce que - Dans son équipe... Qu'est-ce qu'on fait?"

Le poids dans le débat de l'expression du ressenti du personnage de la scène est particulier. Le public semble l'accepter comme un élément d'explication valable et ne le remettra pas en cause, il va l'intégrer dans les discussions qui vont suivre. Par la suite, ce sont les normes collectives, les aspirations professionnelles, le rôle du groupe et les postures managériales qui deviennent des facteurs centraux de la recherche de solutions.

\section{Moyens de faire face à [à la problématique liée aux personnes qui ressentent une pression excessive] par des actions de proximité}

Dans le fil de l'échange, la question du soutien devient donc une voie de solution évidente sur laquelle le public va travailler naturellement, sans prescription directe de l'animateur.

Le public convient que ce soutien peut venir du collectif mais qu'il n'est pas assuré : parfois ce soutien existe, parfois non; cela dépend des collectifs. Le groupe s'accorde néanmoins sur le fait qu'il est important d'avoir des espaces pour pouvoir parler : « rien que d'en parler, et même si on n'a pas les solutions, de partager avec l'équipe de pouvoir dire "vraiment en ce moment, voilà, je suis sous l'eau, j'ai du mal, etc. ». Le fait que ses collègues le sachent, que son manager le sache, enfin c'est une espèce de, finalement, de "je dépose quelque chose ». 
141 La voie du soutien par le manager est aussi explorée. Quelqu'un souligne cependant que si on demande aux managers de systématiser un échange avec leurs collaborateurs sur la charge de travail, ils le feront, dans la mesure où c'est une consigne qu'ils voudront respecter; mais qu'ils risquent de le faire d'une manière qui ne permettra pas d'aller bien loin face aux cas problématiques. Le public convient que la question n'est pas simple et que les managers ne sont pas outillés pour ouvrir vraiment la porte, qu'ils le sont d'autant moins s'ils vivent eux-mêmes les échéances et les attentes de haute performance comme impératives, et s'ils passent beaucoup de temps à rappeler à leur équipe les échéances et les engagements. Le public s'accorde à dire que cette consigne d'apprécier le rapport à la charge de travail sera inefficace sans une formation appropriée des managers. "Moi je l'ai entendu par différents canaux, par les élus, par les salariés, par les managers, euh, personne n'est peut-être suffisamment... aguerri, pour... pour traiter la question de la charge de travail. C'est-à-dire que bon, en effet, quand on dit aux managers, bon voilà vous devez, dans les réunions d'équipe, [...] adresser la question, bon ils le font [...] mais après? C'est, au-delà de cela, bien, c'est difficile pour tout le monde... c'est " comment on pose des questions [...]. Pour adresser ces questions-là, [...] PERSONNE n'est à l'aise avec le sujet, donc, quand tu dis "libérer la parole" c'est, finalement, comment on accompagne à adopter une posture ».

Même si la solution pour le manager n'est pas simple, le collectif note qu'il faut faciliter l'expression parce qu'un effet de soulagement en est attendu, du fait que l'expression de la tension soit accueillie par le manager et l'équipe. Le collectif s'accorde cependant pour dire que cela ne peut suffire.

Un manager témoigne de son expérience. Il a fait intervenir un coach sportif qui a amené l'équipe à discuter les notions de récupération et de repos. Suite à cette intervention, il rapporte avoir instauré des tours d'inclusion et tours de clôture lors de chaque réunion (cf. § 3.2). Il témoigne ensuite qu'une forme de "reprise de contact avec son propre corps", qui lui semble également une bonne chose, même si c'est culturellement décalé. Sera mentionnée également l'importance de travailler la conscience de ses propres limites. L'intervenant qui a parlé du coach souligne qu'il s'agit aussi de faire passer le message de la nécessaire alternance des périodes de forte sollicitation cognitive et émotionnelle avec des périodes de récupération elles aussi intenses [i.e. profondes].

\section{Mise en lien du problème avec des modes d'organisation}

Lors des échanges, plusieurs participants soulignent à quel point l'entreprise véhicule une culture d'engagement : il s'agit d'amener les équipes à s'engager sur des objectifs et des délais, pour laisser au collectif une forte autonomie sur la façon de les atteindre. « Il y a aussi, peut-être [...] culturellement, le fait que, culturellement... Dans la stratégie, on a beaucoup insisté sur "il faut tenir nos engagements, donc cela a été [...] quelque chose de mis [...] TRÈS en avant, et [...] par contre une équipe qui ne TIENT PAS son délai ou son engagement, elle va pas très bien le vivre et je pense qu'elle là, elle ramène le sujet à la maison [...]" ». Dès lors, le risque identifié est clair: si quelqu'un n'y arrive pas, il peut entrer dans une boucle d'autodépréciation, doublée le cas échéant d'une tendance à s'isoler. Les participants s'accordent pour dire que l'entreprise n'est pas réellement armée pour faire face à ce risque. L'hypothèse émerge que la culture de l'engagement deviendrait problématique dès lors qu' « on n'y arrive pas ». Ce peut être vrai pour les individus, mais aussi pour une 
équipe entière qui, à un moment donné, peut « partir en vrille » selon l'expression d'un des participants.

Lors des débats, plusieurs échanges évoquent les méthodes agiles et leur apport potentiel et théorique. Celles-ci permettent (selon certains, en théorie et non en pratique) une proximité forte entre les personnes qui réalisent un développement et le commanditaire interne; si la charge est trop élevée, les processus agiles prévoient une renégociation avec le commanditaire des délais, des objectifs, des moyens. Si c'est bien le principe affiché, dans les faits, il apparaît difficile de dire qu'on était d'accord précédemment sur un objectif mais qu'il faut le redéfinir parce qu'on n'y arrive pas.

De même, dans un cycle agile, est intégrée une période de récupération ("sprint de récupération /respiration »). Dans l'échange, il apparait que c'est surtout théorique et quelqu'un précisera que, selon lui, 4 fois sur 5 , cette période de récupération sert de variable d'ajustement.

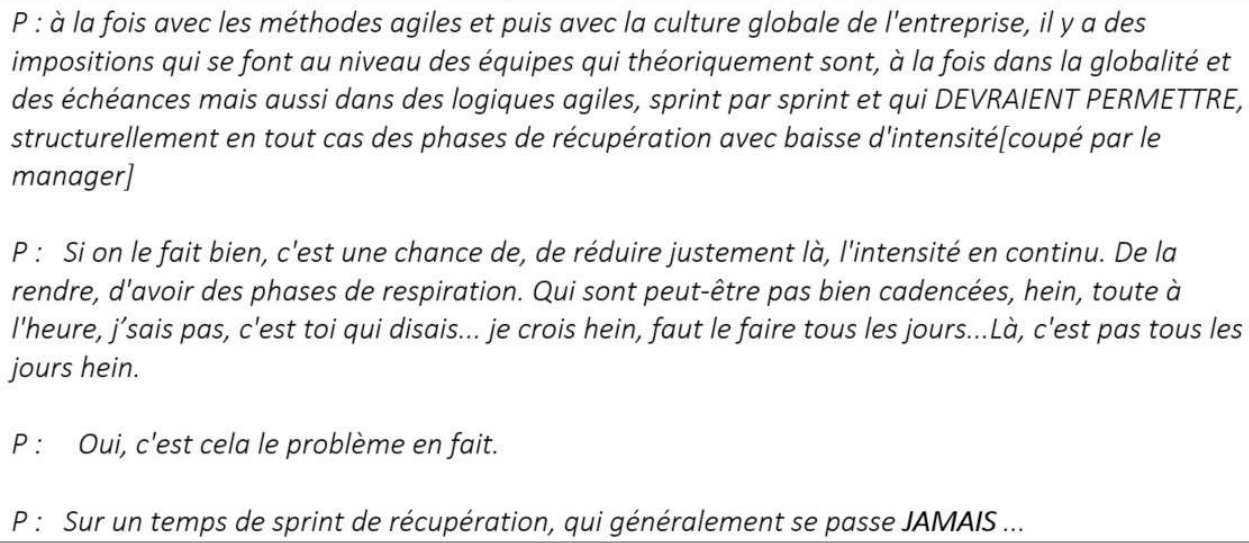

147 On voit dans ces échanges que sont beaucoup soulignés des écarts entre le prescrit et la réalité de l'activité.

\subsection{4. Élaboration d'une solution complexe mise en scène lors d'un remplacement}

Après en avoir exprimé l'idée sur un plan théorique et suite à la sollicitation du joker, une personne du public se propose de remplacer Delphine pour jouer un échange que cette dernière aurait sollicité auprès de son manager, afin d'aborder son sentiment de trop forte charge et de chercher à la fois à alerter et à trouver des solutions qui permettent pour Delphine d'éviter un débordement sur sa sphère privée. En élaborant sa proposition, elle précise que l'échange devrait pour elle se passer en collectif et dit souhaiter expérimenter ce changement pour mieux saisir «les postures qu'on développe de part et d'autre [...] ! Côté manager et côté salarié!»

Deux personnes du public montent sur scène pour prendre des rôles: le rôle de la salariée en difficulté (Delphine) et celui d'un collègue (Valentin). Deux acteurs issus du groupe restreint complètent le casting le premier pour jouer le rôle du manager (Thomas) et l'autre celui d'une seconde collègue (Sylvie).

150 Une fois les acteurs en place, ils improvisent à partir de l'idée proposée. La version jouée met en scène Delphine qui va exprimer lors d'une réunion à quatre le fait qu'elle se sent "sous l'eau». Elle voit dans un premier temps son collègue Valentin et le manager lui rappeler ses objectifs tout en essayant de comprendre la situation. Son autre collègue Sylvie la soutient alors en évoquant un burnout qu'elle dit avoir traversé 
vingt ans plus tôt et en parlant de la gravité du risque. Valentin évoque alors des solutions (structurer les réunions avec des feedbacks sur les ressentis, monitorer mieux son temps de sommeil...) très concrètes et opérationnelles de son point de vue pour prendre du recul et se protéger.

Figure 5 : Mise en scène d'une solution potentielle (remplacement).

Figure 5: Depiction of a potential solution (replacement)

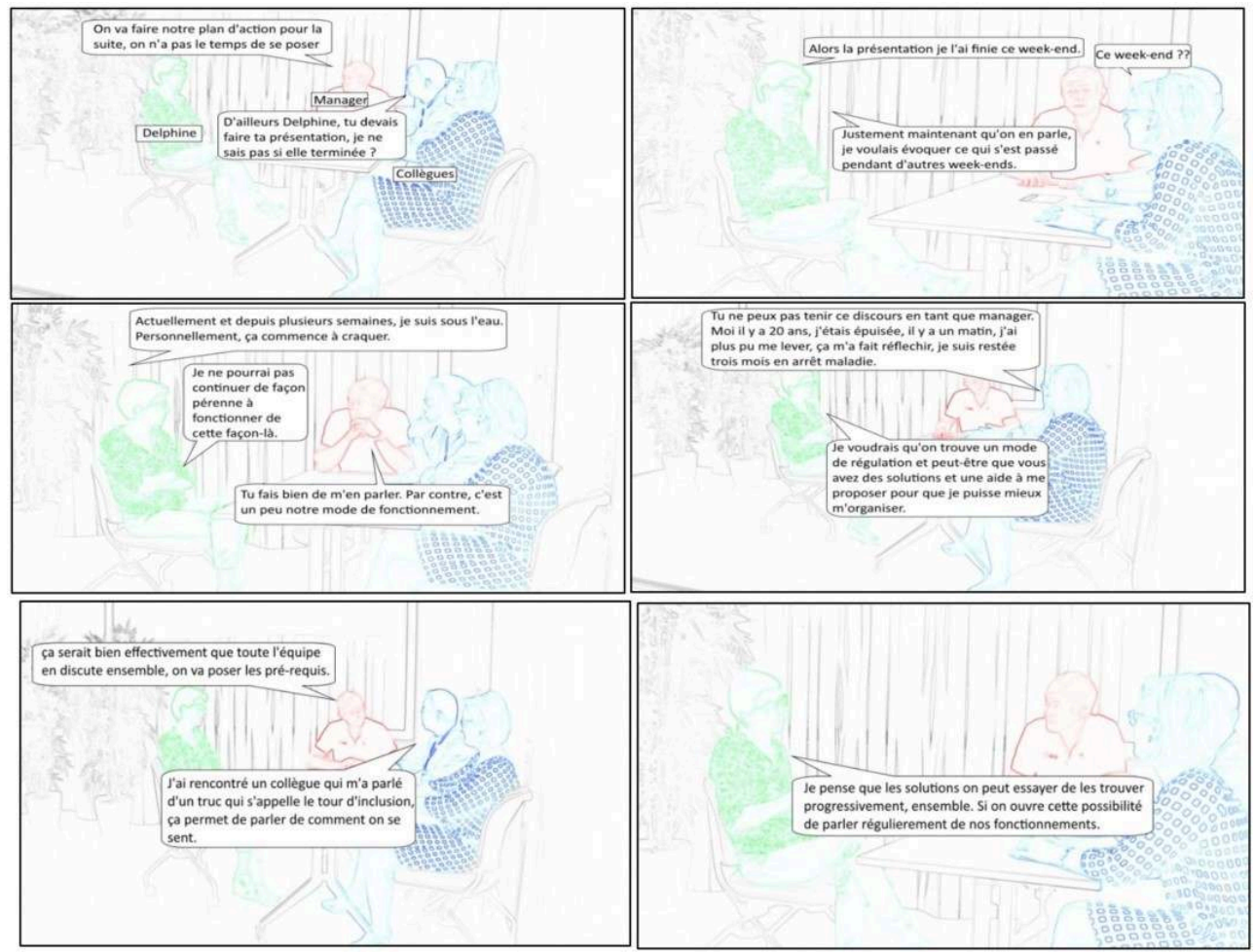

Suite au remplacement, le joker interroge les remplaçants sur leur vécu subjectif au cours de la scène. La personne qui a remplacé Delphine rapporte avoir perçu dans un premier temps qu'elle n'avait qu'une seule alliée et qu'elle s'était dit que ni le manager ni le collègue qui semblaient trouver cela normal ne «lâcheraient ».

Suite à cela, la situation est perçue dans une autre dimension de sa complexité. La scène complémentaire a permis la mise en évidence des difficultés et possibilités du soutien que peuvent apporter des collègues (partage social) et rappelle que des stratégies concrètes permettent une autoprotection ou augmentent la capacité du collectif à anticiper des difficultés.

\section{Discussion}

En choisissant de mobiliser le théâtre-forum pour aborder la problématique du rapport des salariés aux outils numériques, nous sommes partis d'une hypothèse très générale de complexité (Ashby, 1968, 1991; Morin, 2014). Très concrètement, cela signifiait pour nous que le problème était présent transversalement dans l'entreprise (tour le monde est concerné, tant les directions que les opérationnels ou que les managers intermédiaires) et qu'il aurait été erroné de considérer que certains sont victimes des modes d'usage alors que d'autres en sont responsables. Pour nous, cette hypothèse de complexité implique que chacun a une part de responsabilité dans ce qui génère 
frustration et souffrance et chacun peut avoir une part à jouer pour une solution qui gagnerait à être implémentée par tous pour être efficace. L'étude de la littérature sur les implémentations du théâtre-forum en entreprise (Quinlan et al., 2020) montre que, si elles ont porté sur des thèmes très différents (par exemple la violence et le harcèlement au travail), les interventions partagent ce postulat d'une présence du problème dans toutes les strates de l'entreprise et de voies de solution qui demandent à la fois des ajustements comportementaux mis en place par chacun tout autant que des modifications des pratiques organisationnelles, voire une évolution de culture ${ }^{13}$. Tant Quilan que nous-mêmes ou que l'école de sociologie clinique considérons que les problèmes psychosociaux, s'ils sont vécus individuellement, n'en ont pas moins des antécédents structurels et que cette technique permet de mettre en évidence les déterminants liés aux individus comme les causes structurelles profondes en cheminant des premiers vers les seconds et en en pointant les interrelations. En témoignent pour le cas que nous avons développé ici le tableau synthétique présenté dans la figure 4 et le cheminement de l'argumentation que nous avons retracé.

Par ailleurs, la description des intérêts de cette méthode mentionnée par Badache (2019) est pour nous confirmée: les tensions présentées sur scène ont bien été comprises comme des résultantes subjectives - dont la réalité ne peut pourtant être niée - de contraintes objectives générées par des choix de l'organisation. Le public s'est "prêté au jeu» de partir de ces vécus subjectifs pénibles pour remonter aux causes organisationnelles et structurelles de ces tensions, sans nier le rôle que chacun peut jouer à son niveau pour améliorer les choses. En outre, cette posture est en phase avec ce que nous dit Archer et Archer (2003) de la réflexivité. En employant ses mots, on peut dire qu'un des vecteurs de changement implique de passer d'une réflexivité morphostatique, où chacun cherche, sans en avoir réellement conscience, à se conformer à une norme qui n'est pourtant écrite nulle part, à une réflexivité morphogénétique, à partir de laquelle les personnes concernées questionnent leurs pratiques pour les faire évoluer. De plus, les interviews montrent que le questionnement a été individuel en même temps qu'il a été organisationnel.

L'analyse fine des échanges laisse penser que le processus est sinon douloureux, du moins qu'il interpelle émotionnellement. Ce n'est pas simple pour un cadre d'admettre qu'il a pu être en souffrance ou qu'il s'est senti mal du fait de son désir de répondre à la demande d'implication; désir qu'il ressent et qu'il trouve pour partie légitime, pour part excessive. ... et ce n'est pas simple non plus d'opérer ce dévoilement devant un membre de sa direction et devant ses collègues. Quatre salariés l'ont fait, les trois premiers avec beaucoup de retenue («moi perso») et à ce qu'il nous semble, sans vouloir faire porter la responsabilité à quiconque. Le quatrième s'est dévoilé en donnant plus de détails, mais peut-être est-ce parce que « la voie » avait été ouverte par les trois autres? Et encore a-t-il fallu ce qu'on peut considérer comme « une forme de validation » d'un membre du CODIR pour que le débat sur les causes organisationnelles profondes s'enclenche pour ne plus s'interrompre. Nous pensons que la dimension ludique et agie facilite cette expression, même s'il faudrait ajouter d'autres analyses de cas pour l'affirmer avec plus d'assurance. Il apparait en tout cas que le protocole proposé et la façon dont il a été implémenté permettent cette libération de la parole. Ce passage par un moment difficile, où les personnes en présence hésitent entre le dévoilement et la retenue, nous semble avoir également été présent au cours des séquences de construction de la scène. Il correspond pour nous à l'hésitation des 
médecins sur la décision de jouer eux-mêmes ou de nous demander de faire appel à des acteurs pour présenter la scène qui leur tenait à cœur. Là aussi, il a fallu un certain courage pour aller vers le dévoilement plutôt que de rester dans la retenue.

Pour ce qui est de la puissance du dispositif à amener à l'élaboration d'une représentation riche du faisceau de causes, une fois cette libération opérée, la figure 4 donne une idée de la diversité des éléments mis en avant. Parmi les critères d'efficacité en termes d'intelligence collective d'un dispositif d'échange, certains auteurs soulignent l'importance de l'expression émotionnelle authentique et de l'écoute de la pluralité des points de vue. Ceci implique que le dispositif établisse un climat de confiance suffisant pour que des aspects gênants pour certaines personnes ou pour l'organisation et ceux qui la représentent soient abordés (Walumbwa, Luthans, Avey, \& Oke, 2011). Encore une fois, il semble que le protocole mis en place l'ait permis.

Notre protocole de recherche ne nous permet cependant pas de dire grand-chose des avantages du dispositif mis en place par rapport à une situation plus classique où les échanges s'opèrent autour d'une table par exemple. Par contre, dans la mesure où le remplacement a permis en quelque sorte de "tester à chaud» une solution d'abord proposée verbalement, on peut néanmoins dire que cela permet d'aller « un pas plus loin » puisque la personne qui a émis l'idée a pu ensuite voir comment les collègues y ont réagi dans des conditions de relative spontanéité. On peut dire la même chose du remplacement testé lors de la séance de préparation. Certaines solutions proposées lors des échanges verbaux ne semblent pas résister à l'épreuve d'un test consistant à l'incarner par le jeu théâtral. Si sur un plan abstrait, la solution parait avoir du sens, une fois jouée, on constate l'ambiguïté des positions organisationnelles qui peuvent en façade afficher une volonté de respect des contraintes sociétales et légales en matière de préservation de la vie personnelle alors que persiste l'attente d'une forte conformité à l'image traditionnelle du cadre dévoué à son entreprise.

On voit que l'intervenant peut accompagner le groupe large comme le groupe restreint dans une réflexion suscitée par la scène incarnée.

Dans le cas du forum avec le groupe large, les échanges semblent procéder par bascules successives, qui correspondent à des moments à partir desquels des interprétations limitées sont discréditées et disparaissent de l'échange pour laisser la place à des explications et des voies de solution plus complexes, plus élaborées. Dans un premier temps, c'est la question de la possible non-pertinence de la scène qui est traitée, puis écartée (lorsque plusieurs personnes évoquent des situations proches qu'elles ont vécues), puis les explications personnalisantes sont mises à mal au profit de solutions plus complexes. On en vient alors à discuter de mesures se situant au niveau organisationnel le plus élevé, nécessitant de travailler sur la culture de performance pour la mettre en balance avec des objectifs de préservation de la santé mentale, posés au niveau des politiques RH de l'entreprise. Nous pensons que ce travail d'approfondissement est facilité par le travail très particulier de l'intervenant, qui à la fois cadre l'expression de tous les points de vue mais rend possibles, voire contraint les échanges pour qu'ils s'acheminent vers la prise de conscience par tous de la complexité du réel figuré par la scène. C'est du moins le postulat apporté à la fois par les tenants des approches systémiques (Althaus et al. 2013) et par les chercheurs qui ont discuté de la posture du joker (Badache, 2019; Guerre, 1999). L'intervenant se positionne en médiateur "neutre et empathique", sans néanmoins être naif sur ce qui fait avancer l'échange et ce qui risquerait de l'enliser. La posture est commune à d'autres modalités 
d'action: elle est très proche de celle recommandée par Barter dans ses cercles restauratifs (Barter, 2012 ; Ortega, Lyubansky, Nettles, \& Espelage, 2016 ; Stottlemyre, 2018). Sans surprise, elle est construite en référence à la communication non violente (CNV) (Guérin, 2009). Dans notre cas, l'intervenant agit de sorte à ce que chaque partieprenante aux échanges se sente entendue et entende les représentations des autres et les accepte au moins dans une certaine mesure pour y juxtaposer des représentations éventuellement plus complexes. Il s'agit d'apposer les points de vue sans les opposer. L'intervention relatée ici montre que cela fonctionne pour aborder de manière constructive des sujets complexes qui questionnent les a priori productivistes de l'entreprise et pour les rapprocher de leurs effets sur la qualité de vie des salariés.

\section{Conclusion}

Le législateur a voulu inciter les entreprises à développer des normes visant à maîtriser les impacts des innovations liées au numérique sur la qualité de vie au travail, et notamment les impacts des nouveaux outils sur les frontières vie professionnelle - vie personnelle (Shettle, 2020). Une approche couramment adoptée pour améliorer les pratiques dans l'entreprise présuppose que des solutions génériques sont applicables pour tous les salariés, qu'il suffirait dès lors d'en débattre avec les représentants des salariés pour aboutir à des solutions satisfaisantes à la fois du point de vue des impératifs liés au travail et de ceux en rapport à la préservation de la sphère privée. C'est de toute évidence une formalisation trop simple du problème, notamment parce que nos rapports au numérique sont traversés d'ambivalence. Le personnage principal de la scène, Delphine, souhaite tout à la fois répondre positivement à la sollicitation WhatsApp de son collègue du comité de direction et être disponible pour son enfant et son conjoint. La manager qui lui répond dans la première scène de remplacement admet que la règle formelle organisant le droit à la déconnexion existe, mais traduit par ses arguments et par sa posture qu'elle la voit comme peu compatible avec des enjeux de carrière. Le dispositif de théâtre-forum permet de donner une visibilité à ces ambivalences et montre à nos yeux à quel point elles font partie de notre humanité et méritent à ce titre d'être pleinement prises en considération lorsque l'objectif est de mettre des outils techniques au service de l'homme et non l'inverse.

\section{BIBLIOGRAPHIE}

Aggarwal, I., \& Woolley, A. (2013). Two perspectives on intellectual capital and innovation in teams: Collective intelligence and cognitive diversity. In Driving the economy through innovation and entrepreneurship (pp. 453-460). Springer.

Alinsky, S. (1971). Rules for Radicals. New York : Random House.

Alinsky, S. (2012). Être radical. Manuel pragmatique pour radicaux réalistes [1971], traduit de l'anglais par Odile Hellier et Jean Gouriou, Bruxelles, Aden, coll. « Grande bibliothèque d'Aden ». 
Althaus, V., Grosjean, V., \& Brangier, É. (2013). La centration sur le processus du changement : l'apport de l'intervention systémique à l'amélioration du bien-être au travail. Activités, 10(1), 127-149. https://journals.openedition.org/activites/607

Archer, M. S. (2012). The reflexive imperative in late modernity. Cambridge University Press.

Archer, M. S., \& Archer, M. S. (2003). Structure, agency and the internal conversation. Cambridge University Press.

Ashby, W. R. (1968). Principles of the self-organizing system. Modern systems research for the behavioral scientist, 108-118.

Ashby, W. R. (1991). Principles of the self-organizing system. In G.J. Klir (Ed), Facets of systems science (pp. 521-536). Springer.

Ashforth, B. E. (1989). The experience of powerlessness in organisations. Organizational Behavior and Human Decision Processes, 43, 207-242

Ashforth, B. E., Kreiner, G. E., \& Fugate, M. (2000). All in a day's work: Boundaries and micro role transitions. Academy of Management review, 25(3), 472-491.

Badache, R. (2015). De l'improvisation à l'écriture. Utilisation de l'écriture dans la méthode du théâtre institutionnel. Vie sociale, (1), 81-87.

Badache, R. (2019). Théâtre-forum et démocratie. Le dispositif d'atelier citoyen. [Theatre-forum and democracy. The civic workshop scheme]. Connexions, 111(1), 109-118. https://doi.org/ 10.3917/cnx.111.0109

Barter, D. (2012). Walking toward conflict. Tikkun, 27(1), 21-70.

Begon, E., \& Mairesse, P. (2013). Réflexivité et capabilité : la part de création dans l'activité. Éducation permanente, 39-52.

Berditchevskaia, A., \& Bertoncin, C. (2021). How to make good group decisions. Retrieved from UK. Boal, A. (1996). Théâtre de l'opprimé, traduit de l'espagnol par Dominique Lémann. Édition La Découverte \& Syros, Paris.

Boal, A., Mellac, R., \& Rigot-Müller, V. (2004). Jeux pour acteurs et non-acteurs : pratique du théâtre de l'opprimé. La Découverte.

Bobillier Chaumon, M.-E. (2017). Du rôle des TIC dans la transformation digitale de l'activité et de la santé au Travail. Revue des Condtions de Travail - Réseau ANACT, 6, 8.

Bonnemain, A. (2019). Affect et comparaison dans le dialogue en autoconfrontation. Activités, 16(1). https://journals.openedition.org/activites/4007

Boudokhane-Lima, F., \& Felio, C. (2015). Les usages professionnels des TIC : des régulations à construire. Communication Organisation, (2), 139-150.

Bulger, C. A., Matthews, R. A., \& Hoffman, M. E. (2007). Work and personal life boundary management: Boundary strength, work/personal life balance, and the segmentation-integration continuum. Journal of occupational health psychology, 12(4), 365.

Cahour, B., \& Grosjean, V. (2021). Accompagner une réflexivité incarnée par une posture spécifique : exemple de l'entretien d'explicitation et du théâtre-forum. Paper presented at the $55^{e}$ Congrès de la SELF, L'activité et ses frontières. Penser et agir sur les transformations de nos sociétés, Paris. 
Casse, C. (2015). Concevoir un dispositif de retour d'expérience intégrant l'activité réflexive collective : un enjeu de sécurité dans les tunnels routiers. Zotero database.

Casse, C., \& Caroly, S. (2017). Les espaces de débat comme méthodologie d'intervention capacitante pour enrichir le retour d'expérience. Activités, 14(2). https://doi.org/10.4000/ activites.3008

Clark, S. C. (2000). Work/family border theory: A new theory of work/family balance. Human relations, 53(6), 747-770.

Claude, M., Pirlot, M. P., Morand, O., \& Grosjean, V. (2018). Pratiques de connexion et état de santé des cadres. Archives des Maladies Professionnelles et de l'Environnement, 79(3), 460.

Clot, Y. (1999). La fonction psychologique du travail. Paris : Presses Universitaires de France.

Clot, Y., Faïta, D., Fernandez, G., \& Scheller, L. (2000). Entretiens en autoconfrontation croisée : une méthode en clinique de l'activité. Perspectives interdisciplinaires sur le travail et la santé, (2-1).

Comer, C. (2017). « On n'est pas là pour casser du mâle ». La politisation versatile des inégalités conjugales dans les groupes d'agricultrices. ["We're not here to bash men"]. Terrains \& travaux, 30(1), 79-99. https://doi.org/10.3917/tt.030.0079

Créno, L., \& Cahour, B. (2016). Les cadres surchargés par leurs emails : déploiement de l'activité et expérience vécue. Activités, 13(1). https://journals.openedition.org/activites/2698

de Castro, F. G., \& Guerrero, P. (2013). L'organidrame : un dispositif d'intervention et recherche en sociologie clinique. In V. de Gaulejac, F. Giust-Desprairies, \& A. Massa (Eds.), La recherche clinique en sciences sociales (pp. 219-236). Érès.

de Gaulejac, V. (2014). Pour une sociologie clinique du travail. La nouvelle revue du travail, (4). de Gaulejac, V., Giust-Desprairies, F., \& Massa, A. (2013). La recherche clinique en sciences sociales. Erès.

De Keyser, V., \& Olivier, M. (1972). Le besoin de cohérence : dimension sociale et individuelle du champ cognitif de l'homme au travail. Le Travail Humain, 35(1), 49-58.

Detchessahar, M., Gentil, S., Grevin, A., \& Stimec, A. (2015). Quels modes d'intervention pour soutenir la discussion sur le travail dans les organisations? Réflexions méthodologiques à partir de l'intervention dans une clinique. @GRH, 16(3), 63. https://doi.org/10.3917/grh.153.0063

El Wafi, W., Brangier, E., \& Zaddem, F. (2016). Usage des technologies numériques et modèles de la perméabilité des frontières entre la vie personnelle et la vie professionnelle. Psychologie $d u$ Travail et des Organisations, 22(1), 74-87.

Edwards, M., \& Blackwood, K. M. (2017). Artful interventions for workplace bullying: exploring forum theatre. Journal of Workplace Learning, 29(1), 37-48.

Freire, P. (2013). Pédagogie de l'autonomie. Ères Ed.

Garrigou, A. (2011). Le développement de l'ergotoxicologie : une contribution de l'ergonomie à la santé au travail. Habilitation à Diriger des Recherches. Ecole doctorale : Sciences Sociales : société, santé, décision. Université Victor Segalen Bordeaux 2.

Ghliss, Y. (2019). Les photo-discours WhatsApp : éléments d'analyse d'une affordance d'une application mobile. Corela. Cognition, représentation, langage (HS-28).

Ginisty, B. (1977). Saul Alinsky, Manuel de l'animateur social, Coll. Esprit, 1976. Sociologie du travail, 19(19), 205-207. 
Glass, J. (2000). Envisioning the integration of family and work: Toward a kinder, gentler workplace. Contemporary Sociology, 29(1), 129-143.

Grosjean, B. (1999). Le théâtre dans la prévention : intérêt et limites. Lettres du CRIPS, 9.

Guérin, V. (2009). Le théâtre-forum : une approche de développement relationnel. In V. Guérin, \& J. Verber (Eds.), Le monde change... et nous ! Clés et enjeux du développement relationnel (pp. 227-246). Lyon : Chronique Sociale.

Guerre, Y. (1999). Le théâtre-forum. Pour une pédagogie de la citoyenneté. L'Harmattan.

Haddon, L., \& Silverstone, R. (2000). Information and communication technologies and everyday life: Individual and social dimensions. The information society in Europe: Work and life in an age of globalization, 233-257.

Hamel, S. (2012). Le Théâtre-forum : pour une délibération sous le signe de la reconnaissance? Thèse, Université de Montréal,

Hill, E. J., Ferris, M., \& Märtinson, V. (2003). Does it matter where you work? A comparison of how three work venues (traditional office, virtual office, and home office) influence aspects of work and personal/family life. Journal of Vocational Behavior, 63(2), 220-241.

Jauréguiberry, F. (2014). La déconnexion aux technologies de communication. Réseaux, (4), 15-49. Lachaux, J.-P. (2015). Le cerveau funambule : comprendre et apprivoiser son attention grâce aux neurosciences. Paris : Odile Jacob.

Le Moigne, J.-L. (2008). Edgar Morin, le génie de la Reliance. Synergies monde, 4(1), 178-184.

Lebeau, G. (2017). Colère de flic. Paris : Flamarion.

Leimeister, J. M. (2010). Collective intelligence. Business \& Information Systems Engineering, 2(4), 245-248.

Lénel, P. (2011). Théâtre de l'opprimé et intervention sociale. Aux sources de l'éducation populaire ? [Theatre of the Oppressed and Social Intervention]. Agora débats/jeunesses, 58(2), 89-104. https://doi.org/10.3917/agora.058.0089

Leung, L. (2011). Effects of ICT connectedness, permeability, flexibility, and negative spillovers on burnout and job and family satisfaction. Human Technology: An Interdisciplinary Journal on Humans in ICT Environments, 7(3), 250-267.

Leung, L., \& Zhang, R. (2017). Mapping ICT use at home and telecommuting practices: A perspective from work/family border theory. Telematics and Informatics, 34(1), 385-396.

Linhart, D. (2019). Modernisation managériale : tout plutôt qu'une démocratisation du travail. In Manuel indocile de sciences sociales (pp. 832-840). Paris : La Découverte.

Mack, M. (2003). Pleine valeur : pour que l'entreprise génère un nouvel épanouissement économique et humain. Insep Éditions.

Malone, T. W., Atlee, T., \& Lévy, P. (2008). Collective intelligence. Social Text, available at http:// www.socialtext.netlmit-cci-hcilindexcgi

Mazmanian, M., Orlikowski, W. J., \& Yates, J. (2013). The autonomy paradox: The implications of mobile email devices for knowledge professionals. Organization science, 24(5), 1337-1357.

Middleton, C. A., \& Cukier, W. (2006). Is mobile email functional or dysfunctional? Two perspectives on mobile email usage. European Journal of Information Systems, 15(3), 252-260. 
Morand, O. (2020). Mise en débat des pratiques liées à la connexion et à la surconnexion. Thèse de doctorat, Télécom Paris IPP, Paris.

Morand, O., Cahour, B., Bobillier-Chaumon, M.-E., \& Grosjean, V. (2018). Overload of Technological Connections for Communicating at Work. Paper presented at the Congress of the International Ergonomics Association.

Morand, O., Cahour, B., Chaumon, M.-E. B., \& Grosjean, V. (2018). Enjeux et impacts de la connexion au travail ; régulations individuelles et collective. AIPTLF, Bordeaux, juillet.

Morin, E. (2014). Introduction à la pensée complexe. Paris : Le Seuil.

Morin, E., \& Le Moigne, J.-L. (1999). L'intelligence de la complexité. Paris : L'Harmattan.

Morvan, A. (2011). Pour une éducation populaire politique. A partir d'une recherche-action en Bretagne. Thèse de Sciences de l'éducation, sous la direction de Jean-Louis Le Grand, Paris 8.

Murty, P., Paulini, M., \& Maher, M. L. (2010). Collective intelligence and design thinking. Paper presented at the DTRS'10: Design Thinking Research Symposium.

Nam, T. (2014). Technology use and work-life balance. Applied Research in Quality of Life, 9(4), 1017-1040.

O’Hara, K. P., Massimi, M., Harper, R., Rubens, S., \& Morris, J. (2014). Everyday dwelling with WhatsApp. Paper presented at the Proceedings of the $17^{\text {th }}$ ACM conference on Computer supported cooperative work \& social computing.

Olfa Zaïbet, G. (2007). Vers l'intelligence collective des équipes de travail : une étude de cas. Management \& Avenir, 14(4), 41-59. https://doi.org/10.3917/mav.014.0041

Ortega, L., Lyubansky, M., Nettles, S., \& Espelage, D. L. (2016). Outcomes of a restorative circles program in a high school setting. Psychology of Violence, 6(3), 459.

Padis, M.-O. (2008). Obama, de Chicago à Washington. [Obama, from Chicago to Washington]. Esprit, Décembre (12), 6-12. https://doi.org/10.3917/espri.812.0006

Petit, J., \& Dugué, B. (2011). L'intervention ergonomique sur les risques psychosociaux dans les organisations : enjeux théoriques et méthodologiques. [Ergonomic Interventions on Psychosocial Risks in Firms: Theoretical and Methodological Opportunities]. Le Travail Humain, 74(4), 391-409. https://doi.org/10.3917/th.744.0391

Prost, M., \& Zouinar, M. (2015). De l'hyper-connexion à la déconnexion : quand les entreprises tentent de réguler l'usage professionnel des e-mails. Perspectives interdisciplinaires sur le travail et la santé, 17(1).

Quinlan, E., Robertson, S., Urban, A.-M., Findlay, I. M., \& Bilson, B. (2020). Ameliorating Workplace Harassment among Direct Caregivers in Canada's Healthcare System: A Theatre-Based Intervention. Work, Employment and Society, 34(4), 626-643.

Rui, S. (2018). « Réflexivité ». In S. Paugam, Sociologie. Les 100 mots de la sociologie. PUF.

Schön, D. A. (1994). Le praticien réflexif : à la recherche du savoir caché dans l'agir professionnel, traduit et adapté par J. Heynemand et D. Gagnon. Montréal : Les Éditions Logiques (publié originellement en langue anglaise par Basic Books Inc., 1983, sous le titre The reflexive practitioner: How Professionals Think in Action).

Secundo, G., Dumay, J., Schiuma, G., \& Passiante, G. (2016). Managing intellectual capital through a collective intelligence approach. Journal of Intellectual Capital, 17(2), 298-319. 
Shettle, J. (2020). Actualité juridique : Le droit à la déconnexion. Hygiène et Sécurité du Travail, 258, 12-14.

Stottlemyre, N. (2018). Descriptions of Facilitator Practices in Authentic Restorative Processes: A Qualitative Exploration. Capella University,

Sullivan, J., \& Lloyd, R. S. (2006). The forum theatre of Augusto Boal: a dramatic model for dialogue and community-based environmental science. Local Environment, 11(6), 627-646.

Thornton, B., Faires, A., Robbins, M., \& Rollins, E. (2014). The mere presence of a cell phone may be distracting. Social Psychology, 45(6), 479-488.

Valcour, P. M., \& Hunter, L. W. (2005). Technology, Organizations, and Work-Life Integration. In E.E. Kossek, \& S.J. Lambert (Eds.), Organizational, cultural, and individual perspectives (pp. 61-84). LEA. Varela, F. J., Thompson, E., \& Rosch, E. (2016). The embodied mind: Cognitive science and human experience. MIT press.

Wajcman, J., Bittman, M., \& Brown, J. E. (2008). Families without borders: Mobile phones, connectedness and work-home divisions. Sociology, 42(4), 635-652.

Wajcman, J., \& Rose, E. (2011). Constant connectivity: Rethinking interruptions at work. Organization Studies, 32(7), 941-961.

Van Belleghem, L. (2015). «Une ingénierie de la discussion? Chiche!». Actes du 50e congrès international de la Société d'ergonomie de langue française, 23-25 sept. 2015

Van Belleghem, L. (2016). Eliciting activity: a method of analysis at the service of discussion. Le Travail Humain, 79(3), 285. https://doi.org/10.3917/th.793.0285

Van Belleghem, L. (2018). La simulation de l'activité en conception ergonomique : acquis et perspectives. Activités, 15(1). http://doi.org/10.4000/activites.3129

Walumbwa, F. O., Luthans, F., Avey, J. B., \& Oke, A. (2011). Retracted: Authentically leading groups: The mediating role of collective psychological capital and trust. Journal of Organizational Behavior, 32(1), 4-24.

\section{NOTES}

1. Thèse de Doctorat d'Ophélie Morand (2020) financée par l'INRS (tuteur V.Grosjean), codirecteurs B. Cahour et M.-E. Bobillier-Chaumon.

2. Ou virtuellement, dans le cas du télétravail.

3. Les auteurs parlent $d$ '«anytime anywhere responsiveness" ou "anytime, anywhere connectivity » pour définir cette contrainte implicite.

4. Le plus connu des community organizers américains, formé lui aussi dans les quartiers défavorisés de Chicago, est sans doute Barack Obama (Padis, 2008).

5. Ce qui n'implique pas nécessairement qu'ils en aient conscience au départ.

6. Trois séquences de 4 à 5 jours de formation auprès d'un organisme spécialisé, répartis sur deux ans et demi.

7. Deux saynètes ont été préparées, une a été choisie par le groupe pour être présentée au collectif large.

8. Voici ce qu'en dit par exemple Bernard-Marie Chiquet «le tour d'inclusion donne l'occasion pour chacun d'être présent. Il concerne une seule personne à la fois, chacun à son tour et c'est l'occasion de dire ce qui capte votre attention, ou à l'inverse ce qui pourrait vous inciter à être distrait, ce qui vous tient à cour pour l'instant, dans quel état d'esprit vous vous trouvez, et de juste l'exprimer. Cela permet d'être plus 
conscient de ce qui vous distrait ou vous rend présent, et de l'exprimer, de le dire, ce qui a pour effet de vous en détacher, de lâcher prise par rapport à cela. Et d'être plus présent pour la réunion. Donc je vais commencer et on va ensuite faire le tour de table. Pas de réaction des autres, juste une personne à la fois. » 9. Qu'avez-vous appris - Qu'avez-vous apprécié ? Et à l'inverse qu'est-ce qui vous a déplu ? Qu'avez-vous retenu? - Quels éléments pourrions-nous améliorer? - Quels sont les apports selon vous de cette méthode pour transformer les pratiques en entreprise?

10. Qu'est-ce qui vous a amené à participer à cette séance? Comment avez-vous vécu la séance? (Si vous avez remplacé, parlez-en aussi). Qu'avez-vous appris, compris, retenu sur la thématique abordée aujourd'hui? Quels éléments faudrait-il transformer dans la situation actuelle? Qui devrait prendre en charge ces transformations selon vous?

11. Cf commentaires sur le rôle de cet outil au § 1.2. Dans cette application pour smartphone, les usagers ont la possibilité de créer des groupes, c'est-à-dire des ensembles de personnes qui recevront les messages envoyés à ce groupe par ses membres. Le périmètre d'un groupe WhatsApp étant décidé en toute autonomie par ses membres, ils ne correspondent pas aux entités existant dans l'entreprise.

12. «Ils » renvoie ici aux managers et aux directions.

13. Faute de place, nous ne des avons pas présentés ici. Le lecteur intéressé les trouvera dans (Morand, 2020, p. 273 \& seq.)

\section{RÉSUMÉS}

La surconnexion dont la prégnance s'est vue accrue par l'association de l'utilisation du smartphone et de la messagerie pose problème aux entreprises comme aux salariés, notamment en termes de régulation des frontières entre la sphère privée et la sphère professionnelle et d'articulation vie personnelle /vie professionnelle. Des tentatives de régulation des usages excessifs peuvent prendre la forme d'accords d'entreprise ou s'appuyer sur des stratégies individuelles décidées en toute autonomie par les salariés. Nous explorons ici une troisième voie, la mise en place d'espaces d'échange dans les collectifs de travail pour y débattre des éléments pointés par les travailleurs eux-mêmes comme posant problème. Avec le concours d'un collectif de médecins du travail d'une grande entreprise, un dispositif d'échange sur les pratiques numériques liées au travail s'appuyant sur le théâtre-forum a été mis au point et testé. À partir de l'analyse détaillée d'un cas d'usage, l'article examine en quoi la méthode permet à un collectif d'aboutir à une construction enrichie d'une problématique complexe, puis de "porter le débat » dans un cadre plus large incluant des décideurs où sont envisagées des voies de solution. Les résultats attestent des apports du dispositif à la fois pour le groupe de personnes qui ont souhaité interpeller plus largement sur l'importance du problème et pour les membres de l'entreprise qui ont été secondairement interpellés.

E-mail overload, a problem heightened by the combination of the smartphone and messaging, has various negative consequences, particularly in terms of work-life balance. Attempts to find solutions can take the form of company agreements or rely on individual strategies developed by the jobholders themselves. In this article, we explore a third solution, the collective discussion of what the workers themselves feel to be 'problematic'. With the help of a group of occupational physicians in a large company, a debate based on forum-theatre was developed and tested. Based on a thorough analysis of this case study, the article examines how the deployed method enabled 
a group of people that included managers to build a deep understanding of a complex problem and its possible solutions. The results show the benefits of the forum-theatre framework both for the group of people who wished to raise awareness of the importance of the problem and for the company members who were secondarily challenged by this issue.

\section{INDEX}

Keywords : e-mail overload, ICT, forum-theatre, group dynamics, collective intelligence, empowerment

Mots-clés : hyperconnexion, TIC, régulation, théâtre-forum, dynamique de groupe, intelligence collective, pouvoir d'agir

\section{AUTEURS}

\section{VINCENT GROSJEAN}

INRS, rue du Morvan, 54519 Vandœuvre-lès-Nancy

vincent.grosjean@inrs.fr

\section{OPHÉLIE MORAND}

INRS, rue du Morvan, 54519 Vandœuvre-lès-Nancy

I3 Télécom Paris IPP et CNAM Chaire de Psychologie du Travail

ophelie.morand@inrs.fr

\section{BÉATRICE CAHOUR}

CNRS i3 Ecole sup.Télécom Paris / IPP

19 place Marguerite Perrey, 91120 Palaiseau

beatrice.cahour@telecom-paris.fr

\section{MARC-ÉRIC BOBILLIER-CHAUMON}

CNAM Chaire de Psychologie du Travail

41 rue Gay-Lussac, 75005 Paris

marc-eric.bobillier-chaumon@lecnam.net 